This is the author's post-print version of a manuscript accepted for publication in Management Science. This version does not include post-acceptance editing and formatting. Readers who wish to access the published version of this manuscript should go to https://doi.org/10.1287/mnsc.2015.2312. Those who wish to cite this manuscript should cite the published version.

\title{
Optimizing the Deployment of Public Access Defibrillators
}

\author{
Timothy C.Y. Chan, Derya Demirtas, Roy H. Kwon \\ Department of Mechanical and Industrial Engineering, University of Toronto, Toronto, ON M5S 3G8, Canada \\ tcychan@mie.utoronto.ca, demirtas@mie.utoronto.ca, rkwon@mie.utoronto.ca
}

\begin{abstract}
Out-of-hospital cardiac arrest is a significant public health issue and treatment, namely, cardiopulmonary resuscitation and defibrillation, is very time-sensitive. Public access defibrillation programs, which deploy automated external defibrillators (AEDs) for bystander use in an emergency, reduce the time to defibrillation and improve survival rates. In this paper, we develop models to guide the deployment of public AEDs. Our models generalize existing location models and incorporate differences in bystander behavior. We formulate three mixed integer nonlinear models and derive equivalent integer linear reformulations or easily computable bounds. We use kernel density estimation to derive a spatial probability distribution of cardiac arrests that is used for optimization and model evaluation. Using data from Toronto, Canada, we show that optimizing AED deployment outperforms the existing approach by $40 \%$ in coverage and substantial gains can be achieved through relocating existing AEDs. Our results suggest that improvements in survival and cost-effectiveness are possible with optimization.
\end{abstract}

Key words: facility location; coverage models; kernel density estimation; automated external defibrillator; cardiac arrest

\section{Introduction}

Out-of-hospital cardiac arrest (OHCA) is a significant public health issue, responsible for approximately 400,000 deaths annually in North America (Go et al. 2013, Heart and Stroke Foundation of Canada 2013). Cardiac arrest occurs when the heart stops pumping blood in a coordinated fashion due to abnormal heart rhythms. The likelihood of survival from cardiac arrest decreases by 7 to $10 \%$ for every minute of delay in treatment (Larsen et al. 1993, Valenzuela et al. 1997). In fact, less than $5 \%$ of cardiac arrest victims survive to hospital discharge (Nichol et al. 2008). It has been shown that the likelihood of survival can be substantial (50-75\%) with early cardiopulmonary resuscitation (CPR) and a defibrillatory shock to the heart (Valenzuela et al. 2000, Page et al. 2000, Caffrey et al. 2002). While emergency medical services (EMS) personnel are usually the first responders to a cardiac arrest emergency, they often do not arrive in time to save the patient. This motivates the integration of bystanders into the emergency response system by training and 
encouraging them to provide timely CPR and defibrillation in a public cardiac arrest emergency. To facilitate this integration, public access defibrillation (PAD) programs aim to place automated external defibrillators (AEDs) in public locations for bystander use.

An AED is a portable electronic device with vocal and visual prompts that automatically diagnoses cardiac rhythms and delivers a shock to correct abnormal activity in the heart if needed. AEDs are safe, easy to use, and can be used effectively by lay responders with little or no training. In fact, it has been shown that untrained sixth grade students can use them almost as well as trained paramedics (Gundry et al. 1999). Even though PAD programs are found to be associated with markedly improved survival rates (Aufderheide et al. 2006, Hazinski et al. 2005, Hallstrom et al. 2004), only a small percentage of OHCA victims have an AED applied before EMS arrival (Culley et al. 2004). Many factors may contribute to the low usage rate of AEDs, including lack of public awareness, lack of bystander willingness, and lack of data-driven guidance in choosing AED locations. The latter is our focus in this paper.

In North America, the American Heart Association (AHA) and the Heart and Stroke Foundation of Canada (HSFC), working with local PAD programs, assist communities in obtaining AEDs. Current AHA guidelines suggest placing AEDs in locations with one or more cardiac arrests in the last five years, which is cost prohibitive. In practice, priority locations for AED deployment are typically public venues such as schools, convention centers, community centers, and international airports. However, these placements are often made without knowledge of the actual cardiac arrest risk. For instance, in Toronto, the vast majority of registered AEDs are placed in elementary and secondary schools, even though the relative risk of cardiac arrest in these locations is low (Brooks et al. 2013). Furthermore, government funding for public AEDs is often targeted to specific venues that are deemed appropriate by the government but are not necessarily the riskiest.

In this paper, we develop a comprehensive modeling framework to support data-driven decision making in the deployment of public location AEDs, with the ultimate goal of increasing the likelihood of AED usage in a cardiac arrest emergency. We begin by developing three optimization models that consider probabilistic coverage of cardiac arrests using AEDs and address specific, real-life scenarios about AED retrieval and usage. Model 1 considers the scenario where multiple lay responders at the scene of a cardiac arrest fan out and search for nearby AEDs independently. Models 2 and 3 examine the cases when there is only one lay responder available, who retrieves the furthest and closest AED, respectively. We develop a general optimization framework that encompasses all three models, which are presented as mixed integer nonlinear programs. Next, we estimate a geographical distribution of cardiac arrest risk using historical cardiac arrest data and kernel density estimation. Our framework uses the estimated distribution to generate both a "training set" of cardiac arrests to be used as input to the optimization models, as well as 
"testing sets" of cardiac arrests to be used to evaluate the optimization output. This framework is the first to marry both the demand and supply sides of the AED location problem. Lastly, we demonstrate the value of applying our framework using data from Toronto, Canada, and show that significant monetary savings and improvements in cardiac arrest coverage may be possible through optimization. Proofs can be found in the Appendix.

\subsection{Relevant Literature}

Most of the research on AED location has been conducted in the medical community in the last 15 years. One of the earliest efforts categorized buildings in Seattle, Washington and determined the frequency of cardiac arrests that occur in each building type (Becker et al. 1998). Similar studies were conducted in Kansas City, Missouri (Gratton et al. 1999), Windsor, Canada (Fedoruk et al. 2002), Göteborg, Sweden (Engdahl and Herlitz 2005), and Copenhagen, Denmark (Folke et al. 2009). Many of these studies identified facilities having large daily flows of people, such as transportation hubs and shopping malls, as high risk. Fedoruk et al. (2002) and Gratton et al. (1999) also found that casinos are high risk for cardiac arrests. Interestingly, it was shown that placing AEDs in casinos markedly increased the survival rate from OHCA (Valenzuela et al. 2000), primarily due to faster response times made possible by the surveillance systems in place for casino security. In general, these studies suggest that ranking locations based on their risk of cardiac arrest is an appropriate method to guide the placement of public AEDs. However, there are several limitations to this approach. First, identification of high risk locations is highly dependent on the demographics and infrastructure of the studied cities and therefore not generalizable. For instance, while Becker et al. (1998) found a relatively high incidence of cardiac arrest at golf courses, Engdahl and Herlitz (2005) listed golf courses among the low-incidence sites. Second, a substantial number of cardiac arrests happen outdoors and cannot be categorized under any building type. Lastly, a high risk building type may have a large number of constituent facilities spread across the city, making broad AED deployment in this building type cost prohibitive. Mell and Sayre (2008) analyzed the "fire extinguisher model" of putting an AED next to each fire extinguisher for the purpose of wide distribution and increased awareness, but concluded that this approach is not cost-effective. These findings emphasize the need for a generalizable model to guide cost-effective AED deployment.

Researchers have also studied the geographical distribution of cardiac arrest risk. Soo et al. (2001) analyzed OHCA incidents in electoral districts of Nottinghamshire, United Kingdom and found that districts with higher material deprivation scores (Townsend 1987) have significantly higher incidence rates. Sasson et al. (2012) analyzed census tracts in Columbus, Ohio to identify those with both high OHCA risk and low bystander CPR rates. Lerner et al. (2005) used kernel analysis to identify OHCA clusters in Rochester, New York. In general, these studies conclude 
that spatial methods can identify areas for targeted resource allocation, such as identifying the most appropriate areas for community CPR training or AED placement. These studies focus on determining cardiac arrest risk and do not integrate their results within a prescriptive framework for AED deployment.

Optimization has been used extensively to model location problems (Daskin 2008, Fallah et al. 2009, Daskin and Dean 2005) and has been the predominant method for solving emergency-related facility location problems for decades. One of the earliest studies in this area used a set covering model to locate emergency service facilities (Toregas et al. 1971). In a limited resource environment, the maximal covering location problem (MCLP) is a natural alternative (Church and ReVelle 1974). Extensions beyond the classical MCLP have considered expected coverage based on the probability of facility availability (Daskin 1983), survival-based objective functions (Erkut et al. 2008), covering a demand point multiple times (Gendreau et al. 1997), and incorporating a decaying coverage function (Church and Roberts 1983, Karasakal and Karasakal 2004, Berman et al. 2003).

A decaying coverage function is relevant for the AED location problem because, realistically, AED coverage decreases as the distance to the patient increases. However, previous models do not adequately address other important aspects of the AED location problem. First, previous models assume that the decision maker has perfect information on locations of the facilities and that the demand is met by the closest non-busy facility. While this may be reasonable for other location problems, lay responders in a cardiac arrest emergency do not necessarily know where AEDs are located. They need to search for them with the guidance of a 911 operator and available signage. This motivates us to develop models for best-case and worst-case AED retrieval scenarios.

Second, in classical covering models it is assumed that each demand point is serviced by only one facility. Even if there exist back-up facilities in case a preferred one is busy, demand is still met by a single facility at the time of service. In the case of a cardiac arrest, it is desirable to send out many lay responders to look for AEDs, which would increase the likelihood of bringing an AED to the victim. Therefore, we develop a multiple-responder model that maximizes coverage when more than one AED can contribute to the coverage of a cardiac arrest. A similar model minimizing the sum of probabilities of non-coverage, referred to as the "probabilistic partial set covering problem" was introduced by Sherali et al. (1991). They assumed all probabilities were strictly less than one and provided bounds using a branch-and-bound procedure. Lee et al. (2006) removed the restriction on the probabilities and solved cases up to 100 potential facility locations and 200 customers using constraint generation. In this paper, we introduce simple yet effective bounding strategies based on reformulations and solve problems hundreds of times larger in size.

A limited amount of research has been conducted at the intersection of operations research and AEDs. Mandell and Becker (1996) focused on the equitable distribution of AEDs to ambulances 
using a multi-objective integer programming model. Rauner and Bajmoczy (2003) developed a decision model to evaluate the cost-effectiveness of placing AEDs in ambulances. Dao et al. (2011) optimized the locations of AEDs in an indoor environment. Myers and Mohite (2008) used the MCLP to determine locations for AEDs on a university campus. Chan et al. (2013) showed that an MCLP-driven approach to AED deployment outperforms an intuitive population-based method. Chan et al. (2015) developed a robust facility location model for settings in which the location of demand points are uncertain, and demonstrated its application in an AED location problem.

\subsection{Contributions}

We develop the first comprehensive, data-driven framework for public AED deployment, integrating cardiac arrest risk estimation with AED location optimization. Our optimization models generalize the MCLP and represent the first application of a probabilistic coverage concept to AED deployment. We consider three realistic cases of AED retrieval behavior by bystanders and develop corresponding models for AED deployment. The models are mixed integer nonlinear programs, and a contribution of our work lies in the development of mixed integer linear formulation equivalents or tight and easily computable bounds. We derive a theoretical result on the ordering of the optimal objective function values of the three models that enables effective computation of bounds for the multiple-responder case without the need to consider more complicated constraint generation techniques. To the best of our knowledge, this is also the first study to use kernel density estimation to estimate the locations of demand points in facility location optimization. Lastly, we apply our framework to real data to derive several new insights regarding AED deployment. In Toronto, we show that gains in coverage, survival and cost-effectiveness are possible over the status quo by using our framework.

\section{An MCLP Model for AED Location}

We briefly review the MCLP model that our probabilistic coverage models are built upon. Let $I_{e}$ denote the locations of existing AEDs, $I_{c}$ denote the candidate locations for new AEDs, $I=I_{e} \cup I_{c}$, and $|I|=m$. Assume $I_{e} \cap I_{c}=\emptyset$. Let $J$ denote a set of cardiac arrests that are used as the input demand points to be covered $(|J|=n)$. Let $a_{i j}$ be 1 if cardiac arrest $j$ is within the coverage radius of location $i$, and 0 otherwise. We define binary decision variables $x_{j}$ to be 1 if cardiac arrest $j$ is covered, and 0 otherwise; $y_{i}$ to be 1 if an AED is placed at location $i$, and 0 otherwise; and $z_{i j}$ to be 1 if an AED at location $i$ is used to cover cardiac arrest $j$, and 0 otherwise. The MCLP model is:

$$
Z_{M C L P}=\operatorname{maximize} \sum_{j \in J} x_{j}
$$




$$
\begin{array}{ll}
\text { subject to } & \sum_{i \in I_{c}} y_{i} \leq N, \\
& y_{i}=1, \quad \forall i \in I_{e}, \\
& z_{i j} \leq a_{i j} y_{i}, \quad \forall i \in I, j \in J, \\
& x_{j}=\sum_{i \in I} z_{i j}, \quad \forall j \in J, \\
& x_{j}, y_{i}, z_{i j} \in\{0,1\}, \quad \forall i \in I, \in J .
\end{array}
$$

The objective function (1a) maximizes the number of covered cardiac arrests from $J$. Constraint (1b) limits the number of locations in which new AEDs are placed to $N$. Constraint (1c) ensures that the existing AEDs are not moved (a real-world constraint). Constraint (1d) ensures that an AED at location $i$ can only cover cardiac arrest $j$ if $j$ is within the coverage radius of $i$ and an AED is placed at $i$. Lastly, constraint (1e) sets $x_{j}$ to 1 when cardiac arrest $j$ is covered by an AED at some location $i$ and to 0 otherwise. Model (1) is formulated differently than (but is equivalent to) the standard MCLP formulation (Church and ReVelle 1974) to provide a stronger link with the probabilistic coverage models presented in the following section.

Note that the objective function measures "in-sample" coverage - that is, coverage of the demand points that were used as input. However, a decision maker does not know where future cardiac arrests will occur when placing AEDs. Thus, the more relevant measure of coverage that we examine in our computational results is "out-of-sample" coverage - coverage of a separate set of demand points that were not used as input to the optimization model (i.e., different from set $J$ ).

Formulation (1) can be used to estimate the improvement potential of placing additional AEDs, but it does not reflect certain realistic aspects of AED usage. In particular, the MCLP model implicitly assumes that any AED within the coverage radius of a cardiac arrest has the same probability of being used during a cardiac arrest emergency. A more realistic approach is to consider a coverage function that is non-increasing in distance between the AED and the cardiac arrest.

We use the coverage function depicted in Figure 1, which is motivated by "line of sight" and survival curves. We assume that average line of sight is 20 meters and lay responders can immediately spot an AED within this distance. Between 20 and 100 meters, coverage decreases exponentially, mimicking the exponential decrease in survival as a function of time (Valenzuela et al. 1997, Waalewijn et al. 2001, De Maio et al. 2003). Note that we do not assume coverage is equivalent to survival, only that they have similar falloffs with distance/time. Coverage is assumed to be zero beyond 100 meters, which is chosen based on an AHA recommendation for community AED placement (Aufderheide et al. 2006) that advises defibrillation within three minutes of the cardiac arrest victim's collapse. Assuming it takes about 1.5 minutes to set up an AED (Gundry et al. 1999) and a brisk walking speed of 8 kilometers per hour, the maximum distance (round-trip) an 
AED could be transported by a bystander is 100 meters. Formally, the coverage function is defined by

$$
p_{i j}=\left\{\begin{array}{ll}
1, & \text { if } d_{i j} \leq 20, \\
e^{-\alpha\left(d_{i j}-20\right)}, & \text { if } 20<d_{i j} \leq 100, \\
0, & \text { otherwise, }
\end{array} \quad \forall i \in I, \quad \forall j \in J\right.
$$

where $d_{i j}$ is the distance between location $i$ and cardiac arrest $j$ in meters. Nominally we set $\alpha=0.05$ to achieve a reasonably fast decay and a coverage level that is approximately $1 \%$ at 100 meters. We examine the sensitivity of the results to $\alpha$ in Section 6.1.

\section{Figure 1 An exponentially decreasing coverage function.}

\section{Probabilistic Coverage Models for AED Location}

In this section, we develop three formulations for the deployment of AEDs using a decaying coverage function. The objective remains to maximize total coverage, but now each cardiac arrest can be covered "partially", at some value between 0 and 1 . Let $I_{e}, I_{c}, I$ and $N$ be defined as in Section 2 . Let $J$ denote the "training" set of cardiac arrests, sampled from a kernel density estimated distribution of cardiac arrests. We will use the estimated distribution to generate separate out-of-sample "testing" sets to evaluate the optimized AED locations. The process of generating the kernel density estimated distribution is discussed in Section 4 . Let parameter $p_{i j}$ denote the probability (i.e., partial level of coverage) that cardiac arrest $j$ is covered by an AED placed at location $i$. Let $y_{i}$ be 1 if an AED is placed at location $i$, and 0 otherwise. Let $r_{j}$ denote the overall level of coverage provided to cardiac arrest $j$. Furthermore, let variable $z_{i j}$ be the amount of coverage that cardiac arrest $j$ receives from location $i$. The general form of our probabilistic coverage model is:

$$
\begin{array}{ll}
\operatorname{maximize} & \sum_{j \in J} r_{j} \\
\text { subject to } & \sum_{i \in I_{c}} y_{i} \leq N,
\end{array}
$$




$$
\begin{aligned}
& y_{i}=1, \quad \forall i \in I_{e}, \\
& z_{i j}=p_{i j} y_{i}, \quad \forall i \in I, j \in J, \\
& r_{j}=f(\mathbf{z}), \quad \forall j \in J, \\
& 0 \leq r_{j} \leq 1, \quad \forall j \in J, \\
& 0 \leq z_{i j} \leq 1, \quad \forall i \in I, j \in J, \\
& y_{i} \in\{0,1\}, \quad \forall i \in I .
\end{aligned}
$$

The objective function (3a) maximizes total coverage. Constraint (3d) sets $z_{i j}$ to $p_{i j}$ if an AED is placed at location $i$ and 0 otherwise. Constraint (3e) determines the level of coverage $r_{j}$ for cardiac arrest $j$, based on a function, $f(\cdot)$, of the $z_{i j}$ variables.

We believe that formulation (3) captures the essence of the AED deployment problem. In principle, AEDs should be located in such a way as to capture as much continuous spatial demand as possible. Formulation (3) optimizes with respect to a discrete demand distribution that is sampled from the continuous one estimated through kernel density estimation (to be discussed in Section 4.3). The discrete distribution is not "binned" and uses the exact coordinates of the sampled cardiac arrests, so we can use formulation (3) directly. Note that if we interpret $r_{j}$ as a probability of coverage arising from a Bernoulli random variable, then the objective function represents expected coverage for the given set of discrete points $J$ representing the training set.

Depending on the specification of the function $f(\cdot)$, constraint $(3 \mathrm{e})$ provides different ways to calculate the level of coverage provided by the AEDs deployed. The three functions we propose are motivated by three real-world views on AED retrieval during a cardiac arrest emergency, which we describe next.

Model 1 (Multiple-responder): This model is motivated by the scenario where there are many bystanders who witness a cardiac arrest and who then go search independently for an AED to bring back to the victim. Since $z_{i j}$ is defined as the probability that cardiac arrest $j$ is covered by location $i$, we define

$$
f(\mathbf{z})=f^{1}(\mathbf{z}):=1-\prod_{i \in I}\left(1-z_{i j}\right) .
$$

In this case, $r_{j}$ represents the probability that at least one of the bystanders successfully finds and returns with an AED.

Model 2 (Single-responder worst case): This model is motivated by the scenario where there is only one lay responder who is available to retrieve an AED, and who finds the furthest AED from the victim when there is more than one AED nearby. Thus, we define $r_{j}$ to be the worst-case 
probability of covering cardiac arrest $j$ with one of the AEDs that can provide nonzero coverage to cardiac arrest $j$ :

$$
f(\mathbf{z})=f^{2}(\mathbf{z}):= \begin{cases}\min _{i \in I_{j}^{1}} z_{i j}, & \text { if } I_{j}^{1} \neq \emptyset, \\ 0, & \text { otherwise. }\end{cases}
$$

The set $I_{j}^{1}$ denotes the set of locations $i$ in which an AED is placed $\left(y_{i}=1\right)$ and which has $p_{i j}>0$.

Model 3 (Single-responder best case): This model is motivated by the scenario where there is only one lay responder who is available to retrieve an AED, and who finds the closest AED from the victim. Thus, we define $r_{j}$ to be the best-case probability of covering cardiac arrest $j$ :

$$
f(\mathbf{z})=f^{3}(\mathbf{z}):=\max _{i \in I} z_{i j} .
$$

In principle, it is possible to incorporate all three types of behavior into a single model by defining $f(\mathbf{z})$ differently for each $j$, as a weighted combination of $f^{1}(\mathbf{z}), f^{2}(\mathbf{z})$, and $f^{3}(\mathbf{z})$ with weights that depend on $j$. For example, denser areas of the city would have more weight on $f^{1}(\mathbf{z})$. Unfortunately, little is known about actual lay responder behavior in cardiac arrest emergencies and no reliable data is available. Next, we explore the mathematical structure of the three models.

\subsection{Model 1: Multiple-Responder}

Suppose that the number of lay responders is at least as many as the number of AEDs within a certain distance of each cardiac arrest. Using the definition of $f(\mathbf{z})$ in equation (4), Model 1 is:

$$
\begin{aligned}
Z_{1}=\operatorname{maximize} & \sum_{j \in J} r_{j} \\
\text { subject to } & r_{j}=1-\prod_{i \in I}\left(1-z_{i j}\right), \quad \forall j \in J, \\
& (3 \mathrm{~b})-(3 \mathrm{~d}),(3 \mathrm{f})-(3 \mathrm{~h}) .
\end{aligned}
$$

Formulation (7) is nonlinear due to constraint (7b). Next, we derive linear formulations whose optimal values provide bounds on $Z_{1}$.

3.1.1. Bounds Based on Boole and Bonferroni Inequalities: Boole's inequality, also known as the union bound, bounds the probability of a union of events by the sum of the probabilities of the individual events. Since the $z_{i j}$ in formulation (7) model probabilities, we can write the following inequality for each $j$

$$
1-\prod_{i \in I}\left(1-z_{i j}\right) \leq \sum_{i \in I} z_{i j}
$$

and replace constraint $(7 \mathrm{~b})$ with $r_{j} \leq \sum_{i \in I} z_{i j}$ :

$$
\begin{aligned}
\bar{Z}_{1}^{\text {Boole }}=\operatorname{maximize} & \sum_{j \in J} r_{j} \\
& \text { subject to } \quad \\
& r_{j} \leq \sum_{i \in I} z_{i j}, \quad \forall j \in J, \\
& (3 \mathrm{~b})-(3 \mathrm{~d}),(3 \mathrm{f})-(3 \mathrm{~h}) .
\end{aligned}
$$


The optimal value to formulation (8), $\bar{Z}_{1}^{\text {Boole }}$, is an upper bound on $Z_{1}$.

Tighter bounds can be derived by using the Bonferroni inequalities, which generalize Boole's inequality. By the principle of inclusion-exclusion,

$$
1-\prod_{i \in I}\left(1-z_{i j}\right)=\sum_{i \in I} z_{i j}-\sum_{i_{1}<i_{2}} z_{i_{1} j} z_{i_{2} j}+\cdots+\sum_{i_{1}<i_{2}<\cdots<i_{m}}(-1)^{m-1} z_{i_{1} j} z_{i_{2} j} \ldots z_{i_{m} j} .
$$

By truncating the series on the right hand side of (9) at the $t$-th set of summations, the resulting series will be an upper bound (if $t$ is odd) or a lower bound (if $t$ is even) on $1-\prod_{i \in I}\left(1-z_{i j}\right.$ ). Boole's inequality corresponds to $t=1$. As the number of retained summations increases, the bounds become increasingly tight. For $t \geq 2$, the bounds become nonlinear in the decision vector y, but can be linearized by adding auxiliary variables (Adams and Sherali 1986). A lower bound model can be formulated by truncating the series (9) at $t=2$ :

$$
\begin{array}{ll}
\underline{Z}_{1}^{\text {Bonf }}=\operatorname{maximize} & \sum_{j \in J} r_{j} \\
\text { subject to } & r_{j}=\sum_{i \in I} p_{i j} y_{i}-\sum_{i<k} p_{i j} p_{k j} A_{i k}, \quad \forall j \in J, \\
& A_{i k} \leq y_{i}, \quad \forall i \in I, k \in I, i<k, \\
& A_{i k} \leq y_{k}, \quad \forall i \in I, k \in I, i<k, \\
& A_{i k} \geq y_{i}+y_{k}-1, \quad \forall i \in I, k \in I, i<k, \\
& r_{j} \leq 1, \quad \forall j \in J, \\
& 0 \leq A_{i k} \leq 1, \quad \forall i \in I, k \in I, i<k, \\
& (3 \mathrm{~b}),(3 \mathrm{c}),(3 \mathrm{~h}) .
\end{array}
$$

Observe that formulation (10) uses an equality in the constraint defining $r_{j}$, instead of an inequality $(\geq)$, to ensure that $\mathbf{r}$ is appropriately defined by $\mathbf{y}$ in the maximization problem. Accordingly, we remove the constraint $r_{j} \geq 0$ as it may be the case that some of the lower bounds on $r_{j}$ are negative. A tighter lower bound can be obtained by making two modifications. Let $I_{j}^{+}=\left\{i \in I \mid p_{i j}=1\right\}$. First, we modify constraint (10b) to ensure that $r_{j}=1$ when at least one AED is placed close enough to provide full coverage to cardiac arrest $j$ (i.e., $\exists i \in I_{j}^{+}$such that $y_{i}=1$ ). Replacing (10b) with the inequality

$$
r_{j} \leq \sum_{i \in I} p_{i j} y_{i}-\sum_{i<k} p_{i j} p_{k j} A_{i k}+\sum_{i \in I_{j}^{+}} y_{i}, \quad \forall j \in J
$$

prevents the underestimation of $r_{j}$ in the case that $j$ is fully covered. Second, after solving formulation (10) with constraint (11) in place of constraint (10b), we can further improve the bound by simply replacing all negative $r_{j}^{*}$ by 0 . We'll refer to this improved bound as $\underline{Z}_{1}^{\text {Imp-Bonf }}$. Additional 
lower bounds can be derived by simply finding feasible solutions to formulation (7) via other methods. In Section 6.1, we demonstrate that an optimal solution to Model 3 provides a good lower bound on Model 1.

By truncating the series (9) at $t=3$ and using the same linearization technique as above, we can generate a substantially larger optimization problem that improves upon the Boole upper bound. However, our computational results demonstrate that $\bar{Z}_{1}^{\text {Boole }}$ provides a strong and scalable upper bound on $Z_{1}$.

\subsection{Model 2: Single-Responder Worst Case}

The second model is motivated by the scenario where there is only one responder available to retrieve an AED and who finds the furthest AED within a certain distance to bring to the patient. Let $I_{j}^{1}=\left\{i \in I \mid p_{i j}>0\right.$ and $\left.y_{i}=1\right\}, I_{j}^{0}=\left\{i \in I \mid p_{i j}>0\right.$ and $\left.y_{i}=0\right\}$ and $I_{j}=I_{j}^{0} \cup I_{j}^{1}$. The set $I_{j}^{1}$ is the set of locations that can cover cardiac arrest $j$, and in which AEDs are placed. The set $I_{j}^{0}$ is the set of locations that cover cardiac arrest $j$, but which do not have any AEDs placed. These definitions are needed to model the fact that we would like the worst case to be taken over all locations within a certain distance from a given cardiac arrest and for which there is an AED placed. As such, Model 2 has the flavor of a max-min model:

$$
\begin{array}{ll}
Z_{2}=\operatorname{maximize} & \sum_{j \in J} r_{j} \\
\text { subject to } & r_{j}= \begin{cases}\min _{i \in I_{j}^{1}} z_{i j}, & \text { if } I_{j}^{1} \neq \emptyset, \\
0, & \text { otherwise, }\end{cases} \\
& (3 \mathrm{~b})-(3 \mathrm{~d}),(3 \mathrm{f})-(3 \mathrm{~h}) .
\end{array}
$$

Formulation (12) is not linear because of the dependence of the set $I_{j}^{1}$ on the value of $y_{i}$ in constraint (12b). However, we can derive an equivalence between the set of optimal solutions of (12) and the set of optimal solutions to the following mixed integer linear optimization model:

$$
\begin{aligned}
\operatorname{maximize} & \sum_{j \in J} r_{j} \\
\text { subject to } & r_{j} \leq 1-\left(1-p_{i j}\right) y_{i}, \quad \forall i \in I_{j}, j \in J, \\
& r_{j} \leq \sum_{i \in I_{j}} z_{i j}, \quad \forall j \in J, \\
& (3 \mathrm{~b})-(3 \mathrm{~d}),(3 \mathrm{f})-(3 \mathrm{~h}) .
\end{aligned}
$$

Proposition 1. A solution $(\mathbf{r}, \mathbf{z}, \mathbf{y})$ is optimal to formulation (13) if and only if it is optimal to formulation (12).

Thus an optimal solution to the nonlinear Model 2 can be found by solving a mixed integer linear program. 


\subsection{Model 3: Single-Responder Best Case}

The third model is motivated by the scenario where there is only one responder available to retrieve an AED and who finds the closest AED in a certain radius to bring to the patient. Coupled with Model 2, these formulations provide bounds on the range of performance possible when only one person is available to search for an AED in a cardiac arrest emergency. Moreover, solutions to Model 3 are near optimal solutions for Model 1, as will be demonstrated computationally in Section 6 , with theoretical support in Section 3.4. We formulate Model 3 as:

$$
\begin{aligned}
Z_{3}=\operatorname{maximize} & \sum_{j \in J} r_{j} \\
\text { subject to } & r_{j}=\max _{i \in I} z_{i j}, \quad \forall j \in J, \\
& (3 \mathrm{~b})-(3 \mathrm{~d}),(3 \mathrm{f})-(3 \mathrm{~h}) .
\end{aligned}
$$

Although formulation (14) is nonlinear, we derive an equivalent linear formulation and show that it is equivalent to a model in the literature. Let $I_{j}=\left\{i \in I \mid p_{i j}>0\right\}$. Using our notation, consider the gradual covering decay model of Berman et al. (2003) and the maximal covering location model in the presence of partial coverage (MCLP-P) of Karasakal and Karasakal (2004):

$$
\begin{aligned}
\operatorname{maximize} & \sum_{j \in J} \sum_{i \in I_{j}} p_{i j} w_{i j} \\
\text { subject to } & w_{i j} \leq y_{i}, \quad \forall i \in I_{j}, j \in J, \\
& \sum_{i \in I_{j}} w_{i j} \leq 1, \quad \forall j \in J, \\
& w_{i j} \in\{0,1\}, \quad \forall i \in I_{j}, j \in J, \\
& (3 \mathrm{~b}),(3 \mathrm{c}),(3 \mathrm{~h}) .
\end{aligned}
$$

The decision variable $y_{i}$ in formulation (15) has the same interpretation as before (whether or not an AED is placed at location $i$ ). The decision variable $w_{i j}$ is 1 if cardiac arrest $j$ is covered by an AED at location $i$ at some nonzero level (either partially or fully covered by an AED).

Proposition 2. Formulation (14) is equivalent to formulation (15).

Berman et al. (2003) derived formulation (15) from a combinatorial description (instead of an integer programming formulation) of model (14).

\subsection{Relationship Between Models 1, 2, 3 and MCLP}

In this section, we characterize the relationship between the optimal values of Model $1\left(Z_{1}\right)$, Model 2 $\left(Z_{2}\right)$, Model $3\left(Z_{3}\right)$ and the original MCLP model $\left(Z_{M C L P}\right)$. We show that the original MCLP is equivalent to the three probabilistic coverage models if $p_{i j} \in\{0,1\}$. In other words, the three models 
we develop are generalizations of the standard MCLP. Note that for each of the three models, given a feasible $\mathbf{y}$ (one that satisfies $\sum_{i \in I_{c}} y_{i} \leq N, y_{i}=1$ for $i \in I_{e}, y_{i} \in\{0,1\}$ for $i \in I$ ), $\mathbf{r}$ and $\mathbf{z}$ are uniquely determined. Therefore, to compare feasible solutions between formulations, it suffices to consider just $\mathbf{y}$. Furthermore, note that if $\mathbf{y}$ is feasible for one model, it is feasible for all of them.

Proposition 3. Let $Z_{k}^{\mathrm{y}}$ be the objective function value of Model $k, k \in\{1,2,3, M C L P\}$, evaluated using a feasible solution $\mathbf{y}$. Then, $Z_{M C L P}^{\mathbf{y}} \geq Z_{1}^{\mathbf{y}} \geq Z_{3}^{\mathbf{y}} \geq Z_{2}^{\mathbf{y}}$, with equality throughout if $p_{i j} \in$ $\{0,1\}$ for every $i \in I$ and $j \in J$.

THEOREM 1. $Z_{M C L P} \geq Z_{1} \geq Z_{3} \geq Z_{2}$, with equality throughout if $p_{i j} \in\{0,1\}$ for every $i \in I$ and $j \in J$.

The proof of Proposition 3 suggests that an optimal solution $\mathbf{y}^{*}$ to Model 3 provides a good lower bound (i.e., $Z_{1}^{\mathrm{y}^{*}}$ ) on $Z_{1}$. This insight is supported by the computational results.

\section{Spatial Analysis of Cardiac Arrests}

In this section, we first show the stability of cardiac arrest incidence in Toronto, Canada. Then, we estimate a spatial probability distribution of cardiac arrests to be used in our computational experiments.

\subsection{Cardiac Arrest Data}

We obtained cardiac arrest data from the Resuscitation Outcomes Consortium (ROC) EpistryCardiac Arrest database (Morrison et al. 2008). ROC is a large consortium of 11 centers and over 200 emergency medical services operations in North America. We considered 1,310 public atraumatic cardiac arrests that occurred in Toronto between December 16, 2005 and July 15, 2010. Each database entry includes geographical coordinates of the cardiac arrest episode.

\subsection{Spatiotemporal Stability of Cardiac Arrests}

While it is impossible to forecast exactly where future cardiac arrests will occur, it has been shown that the geographical distribution and number of cardiac arrests remains steady over time (Sasson et al. 2010). That study looked at three years of cardiac arrest data and 161 census tracts from Fulton County, Georgia and determined that census tracts have stable rates of cardiac arrest over time using intraclass correlation coefficient (ICC) (Fisher 1921, Shrout and Fleiss 1979). In this context, ICC measures how stable the cardiac arrest count is in each census tract over the three years. We repeated this experiment for Toronto using four years of public cardiac arrest data (20062009) and 140 neighborhoods defined by the City of Toronto. We found that the ICC was 0.72 [95\% CI, 0.66 to 0.78], double the value of 0.36 reported in Sasson et al. (2010), which was considered to be stable. Thus, we believe the spatial demand for AEDs is stable over time in Toronto. 


\subsection{Estimating a Spatial Distribution of Cardiac Arrest Risk}

We use kernel density estimation (KDE), the most commonly used nonparametric approach to density estimation, to estimate a spatial distribution of cardiac arrest risk (Wand and Jones 1995, Scott 2009). Given a set of discrete points, KDE smooths out and aggregates the contributions of each to create a composite density function. The smoothing parameter, the "bandwidth", determines the extent of spread of each point. We applied multivariate KDE to the set of 1,310 historical cardiac arrests and used the method in Botev et al. (2010) to select the appropriate bandwidth. This method improves upon the most popular data-driven bandwidth selection technique, the plugin method (Sheather and Jones 1991), by eliminating the assumption that the data comes from a normal distribution. The two-dimensional bandwidth (east-west, north-south) was found to be 225 meters by 172 meters. Figure 2 shows the resulting spatial distribution in Toronto.

Figure 2 Kernel density estimation of cardiac arrests in Toronto.

\section{Experimental Design}

Data from Toronto, Canada is used in the subsequent computational experiments. This section describes the AED location data and the experimental setup.

\subsection{AED Location Data}

Locations of existing registered AEDs: We obtained a list of 1,669 registered AEDs in Toronto as of September 2009 from Toronto EMS. Toronto EMS aims to register AEDs placed by the several regional PAD programs, as well as AEDs that are privately owned but placed in public settings. The database contains the exact mailing addresses of the buildings in which the AEDs are placed 
and is integrated into a computer-assisted dispatch system used by the 911 operator. However, the current system is set up to alert a 911 operator only if the location of the cardiac arrest is an exact match to a location of an AED in the database. In the absence of an exact address match, it is unlikely that the dispatcher will be able to guide the caller to a nearby AED, even if it is next door. Note that relocating existing AEDs is uncommon and that AED registration is not mandatory in Ontario. While this implies that our list does not contain all AEDs in the public domain, only registered AEDs are likely to be available to lay responders during an emergency.

Candidate locations for new AEDs: We obtained a list of public building and business locations from the City of Toronto's City Planning Division. They conduct an annual Employment Survey that aims to collect data on every business establishment in Toronto. We used the 2009 Employment Survey (City of Toronto 2010), which provided us with geographical coordinates for 25,851 unique public buildings in Toronto. We used these buildings as the set of potential locations for additional AEDs.

Distance calculations: All geographical information and address information was converted into the Universal Transverse Mercator format to facilitate distance calculations. Distances between cardiac arrests and existing or potential AEDs were calculated using the Euclidean metric. Straightline distance is a reasonable approximation to be used in AED location since the coverage radius is small enough that there is often a direct path through buildings or pathways for pedestrians (unlike the road network on which ambulances must travel). Since there is no field in the Epistry database to record floor information, we ignore vertical distance. This issue is discussed further in the next section.

\subsection{Experimental Setup}

We simulated a set of 5,000 cardiac arrests from the distribution in Figure 2 as the input set $J$ for our optimization models (i.e., the "training" set, see Figure 3). The estimated distribution was also used to generate 100 sets of cardiac arrests $J_{1}^{\prime}, \ldots, J_{100}^{\prime}$, which were used to measure the coverage provided by the optimized AED locations (i.e., the "testing" sets). Each of the 100 testing sets had 300 cardiac arrests, the approximate number of public location cardiac arrests that occur in Toronto in one year.

We solved formulation (8) (Boole UB), formulation (10) (Improved Bonferroni LB with equation (11) in place of constraint (10b) and $\mathbf{r}^{*}$ post-processing), formulation (13) (Model 2) and formulation (15) (Model 3) using the exponentially decreasing coverage function in Figure 1. We also solved two MCLP formulations, one with a 100-meter coverage radius and one with a 20 -meter radius, using the more compact formulation of Church and ReVelle (1974). We conducted runs for values of $N$ from 0 to 200. Note that $N$ is not the number of additional AEDs deployed, but 
rather the number of additional locations where AEDs will be deployed. The latter interpretation accounts for the three-dimensional nature of a city, especially in downtown areas where high-rises are abundant. If one of the $N$ locations is a high-rise, we assume that in addition to covering the area surrounding the base of the building according to the coverage function, enough AEDs are installed throughout the building to cover the vertical dimension. Motivated by real-life proposals for AED location inside a building, a weaker assumption could be that an AED is installed in the elevator, which suffices to cover the building both vertically and in the plane.

All formulations were solved using CPLEX 12.1 via AMPL. All models except Model 3 and the Improved Bonferroni LB model were solved using a Linux workstation with a $2.67 \mathrm{GHz}$ quad-core CPU and $6 \mathrm{~GB}$ of RAM. Due to their larger memory requirements, Model 3 and the Improved Bonferroni LB model were solved on a computer cluster using a single Linux node with two 3.07 $\mathrm{GHz}$ 6-core CPUs and 64 GB of RAM. We pre-processed the set of candidate AED locations and removed those that were more than 100 meters from any cardiac arrest in the training set, resulting in 11,701 candidate AED locations. Table 1 compares the size and run time (CPU time) of each formulation.

Optimal AED locations from the models were evaluated using the 100 cardiac arrest testing sets. Statistics such as mean, median, minimum and maximum coverage values were calculated over all testing sets for each model and multiple $N$ values. The overall framework/process flow is depicted in Figure 4.

\section{Results}

\subsection{Comparing Models and Bounds}

Recall that lower bounds on $Z_{1}$ can be obtained via evaluation of feasible solutions obtained through other methods. So in addition to the direct lower bound $\underline{Z}_{1}^{\text {Imp-Bonf }}$, we generated additional lower 
Table 1 Problem sizes for all optimization models using 11,701 AED locations (10,032 candidate, 1,669 existing) and the 5,000 cardiac arrests from the training set.

\begin{tabular}{|c|c|c|c|c|c|}
\hline \multirow{2}{*}{ Problem } & \multirow{2}{*}{$\begin{array}{c}\text { Binary } \\
\text { Variables }\end{array}$} & \multirow{2}{*}{$\begin{array}{c}\text { Continuous } \\
\text { Variables }\end{array}$} & \multirow{2}{*}{ Constraints } & \multicolumn{2}{|c|}{ Run Times } \\
\hline & & & & $(N=\mathbf{5})$ & $(N=\mathbf{2 0 0})$ \\
\hline MCLP & 11,701 & 5,000 & $\sim 28,000$ & 0.07 & 0.11 \\
\hline Boole UB on Model 1 & 11,701 & 5,000 & $\sim 12,000$ & 0.09 & 3.58 \\
\hline Imp. Bonf. LB on Model 1 & 11,701 & $\sim 190,000$ & $\sim 750,000$ & 217.34 & 9003.32 \\
\hline Model 2 & 11,701 & 5,000 & $\sim 28,000$ & 0.29 & 8.67 \\
\hline Model 3 & $\sim 58.5$ million & - & $\sim 58.5$ million & 277.21 & 274.90 \\
\hline
\end{tabular}

Note. Run time $=\mathrm{CPU}$ time in seconds.

Figure 4 Overview of our framework combining optimization models with kernel density estimated cardiac

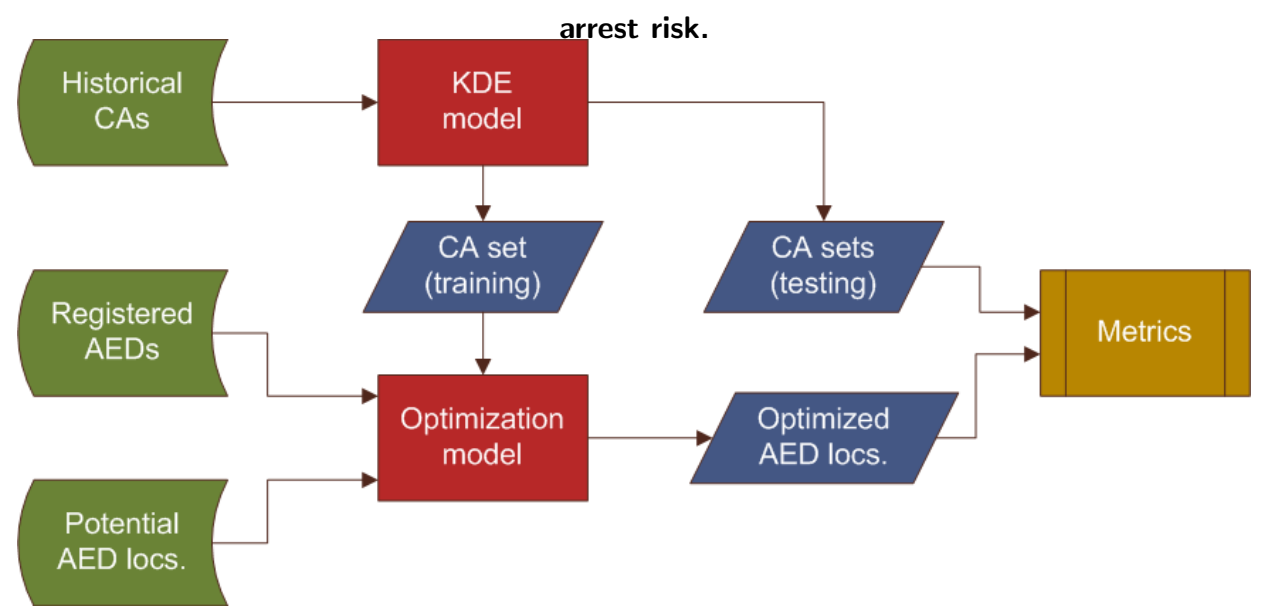

bounds by using the optimal $\mathbf{y}^{*}$ obtained by solving the MCLP with a 20-meter radius, MCLP with a 100-meter radius, Model 2, Model 3, improved Bonferroni LB and Boole UB formulations and substituting them into the objective function of Model 1: $Z_{1}^{\mathbf{y}^{*}}=\sum_{j \in J}\left(1-\prod_{i \in I}\left(1-p_{i j} y_{i}^{*}\right)\right)$.

Table 2 compares the upper and lower bounds on $Z_{1}$ using the set $J$. Note that in this case coverage is evaluated on the training set (not the testing sets) to accurately measure the performance of the bounds. Substituting the solution from the Improved Bonferroni model into the Model 1 objective provides the strongest lower bound. The optimality gap is roughly $5 \%$ over all values of $N$. It is followed closely by the optimal solution to Model 3 (the difference is at most $0.5 \%$ from the Improved Bonferroni model for all values of $N$ ). Since Model 3 is more tractable, we will use its optimal solution to approximate the Model 1 solution going forward. Also note that both MCLP solutions provide poor lower bounds on $Z_{1}$, especially as $N$ increases, reinforcing the need for models that account for probabilistic coverage.

Figure 5 summarizes the performance of each model, optimized using the training set and evaluated using the respective model objective function on the 100 testing sets. Interquartile boxes mark the $25 \%, 50 \%$, and $75 \%$ percentiles; circles mark the means; whiskers indicate min/max values over the 100 test sets. Model 1 has the lowest coefficient of variation among all models. The roughly 
Table 2 Bounds on Model 1 optimized and evaluated using the training set of 5,000 cardiac arrests

\begin{tabular}{|c|c|c|c|c|c|c|c|c|c|}
\hline \multirow{2}{*}{$N$} & Upper Bound & Lower Bound & \multicolumn{5}{|c|}{ Substitution Lower Bounds } & \multirow{2}{*}{ Opt. } \\
\cline { 2 - 8 } & $\bar{Z}_{1}^{\text {Boole }}$ & $\underline{Z}_{1}^{\text {Imp-Bonf }}$ & $Z_{1}^{\mathbf{y}^{M C L P_{20}}}$ & $Z_{1}^{\mathbf{y}^{M C L P 100}}$ & $Z_{1}^{\mathbf{y}^{\text {Boole }}}$ & $Z_{1}^{\mathbf{y}^{\text {Imp-Bonf }}}$ & $Z_{1}^{\mathbf{y}^{2}}$ & $Z_{1}^{\mathbf{y}^{3}}$ & $\mathrm{Gap}^{(\%)}$ \\
\hline \hline 0 & 219.1 & 195.3 & 208.2 & 208.2 & 208.2 & 208.2 & 208.2 & 208.2 & 5.2 \\
10 & 253.3 & 228.0 & 230.3 & 225.0 & 240.4 & 240.9 & 236.7 & 240.8 & 5.1 \\
20 & 279.1 & 252.7 & 257.1 & 234.6 & 265.3 & 265.7 & 258.0 & 265.5 & 5.0 \\
30 & 302.7 & 275.1 & 270.8 & 241.0 & 287.4 & 288.2 & 276.8 & 287.7 & 5.1 \\
50 & 345.0 & 315.5 & 293.8 & 253.6 & 327.8 & 328.6 & 309.5 & 327.8 & 5.0 \\
100 & 430.6 & 396.7 & 350.9 & 292.5 & 407.7 & 410.3 & 373.8 & 409.4 & 5.0 \\
150 & 502.3 & 464.3 & 412.5 & 322.0 & 475.5 & 478.0 & 429.9 & 476.5 & 5.1 \\
200 & 564.7 & 523.0 & 472.6 & 344.0 & 532.1 & 536.9 & 481.0 & 534.3 & 5.2 \\
\hline
\end{tabular}

Shaded cells highlight the best lower bound for the given value of $N$.

$50 \%$ increase in coverage from Model 2 to Model 3 can be thought of as the potential gain that can be achieved by improving AED retrieval efforts. As expected, having additional responders search for AEDs (Model 1) improves overall coverage (12\% compared to Model 3, 69\% compared to Model 2, on average).

Figure 5 Coverage results from Model 1, 2 and 3 on 100 testing sets, each with 300 cardiac arrests

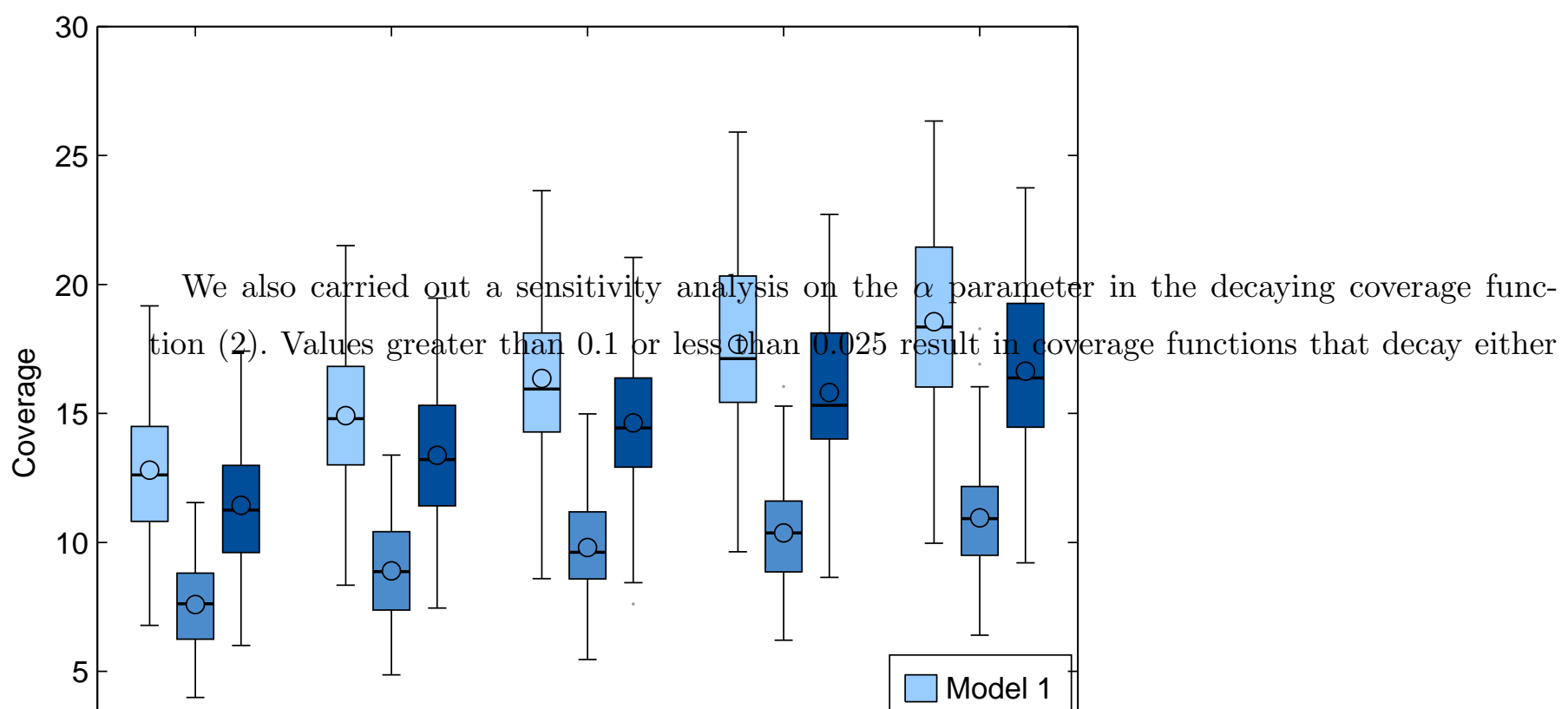


too quickly (under $2 \%$ coverage at about 60 meters) or too slowly (a large drop off in coverage at 100 meters). Thus, we focused on the sensitivity to values of $\alpha$ in the range 0.025 to 0.1 . We did the analysis for $N=50,100,150,200$. We ran the optimization models for four $\alpha$ values, 0.025 , $0.05,0.075$, and 0.1 on the training set, and measured the performance of the resulting optimal locations on the 100 testing sets for each of the four $\alpha$ values (sixteen combinations in total for each value of $N$ ). We found that the choice of $\alpha$ in training does not affect the results by more than $4 \%$ across the $\alpha$ values used in testing. Thus, coverage appears to be robust to misspecification in the input $\alpha$, within the range considered.

\subsection{The Value of Optimizing AED Deployment}

In this section, we quantify the potential gain in coverage from optimizing AED locations according to our models, over the existing approach to AED deployment and two population-guided approaches in the literature.

6.2.1. Existing Approach to AED Deployment: Public AED deployment is largely carried out by individual sites with support from their local PAD program. In Canada, these AEDs are typically purchased using funds provided by the Heart and Stroke Foundation. In Ontario, a committee that includes EMS, city and school board representatives, physicians, researchers, and individuals with public health or sport facility planning expertise reviews applications for AED funding from across the province. As part of the decision making process, the committee considers information about the site such as people flow and the presence of existing AEDs. However, when the funding comes from a specific sponsor (e.g., the government) it is usually targeted at only certain types of facilities; facility-type then becomes the overriding decision criterion in the granting process. While certain high risk sites may be targeted (e.g., sports and recreation centers), many others may be excluded. Note that AEDs funded through this manner represent only a subset of public, registered AEDs. Others may be purchased with private funds but still registered. Thus, we think of the existing approach to AED deployment in Toronto as being partially centralized and partially de-centralized.

6.2.2. Population-Guided Approaches to AED Deployment: We use the two population-guided heuristics developed in Chan et al. (2013) for comparison. Heuristic 1 assigns the estimated daytime population in each census tract to the buildings situated in that census tract, proportional to the number of businesses in each building. Then, all buildings in Toronto are rank ordered based on the assigned population and the top $N$ buildings are selected for AED placement. Heuristic 2 is a variant of Heuristic 1 and assigns the estimated daytime population to buildings proportional to the number of floors, instead of number of businesses, in each building. 
6.2.3. Comparing AED Deployment Approaches: In our experiment, we assume the 1,669 AEDs placed as of September 2009 are fixed. We obtained an updated AED list as of February 2013 that had an additional 403 public, registered AEDs in unique locations compared to the list as of September 2009. We solved the optimization models using the training set $J$ with $N=403$. We also applied the two population-guided heuristics with $N=403$. Then, we evaluated the AED locations from the optimization models, heuristics, and the actual deployment over the 100 testing sets. The average coverage values calculated using the Model 1 objective function are shown in Figure 6. The darkest bars indicate the coverage provided by the existing 1,669 AEDs, while the lighter bars indicate additional coverage provided by 403 "new" AEDs either in addition the existing ones (i.e., additional) or in place of the existing ones (i.e., stand-alone). The additional coverage provided by the optimized AEDs is not only higher than the coverage by the actual AEDs but also higher than the coverage provided by the heuristic approaches. The existing approach to AED deployment gives up about $40 \%$ in coverage compared to the optimization approach. Using a pairwise independent samples $t$-test (coverage values were approximately normally distributed across the 100 testing sets) confirms that there is a significant difference in the coverage values $(p<0.05)$ between the optimized approach and the other approaches. A similar experiment where coverage is measured using Model 2 and Model 3 results in a $52 \%$ and $43 \%$ difference, respectively, between the actual and optimized approaches on coverage, both significant at the $95 \%$ level. Table 3 shows that optimization outperforms the other approaches on a range of coverage metrics including mean, maximum, minimum, $0.1 \mathrm{VaR}$ (10th percentile) and coefficient of variation.

Figure 6 A comparison of coverage from the actual, heuristic, and optimized deployment methods.

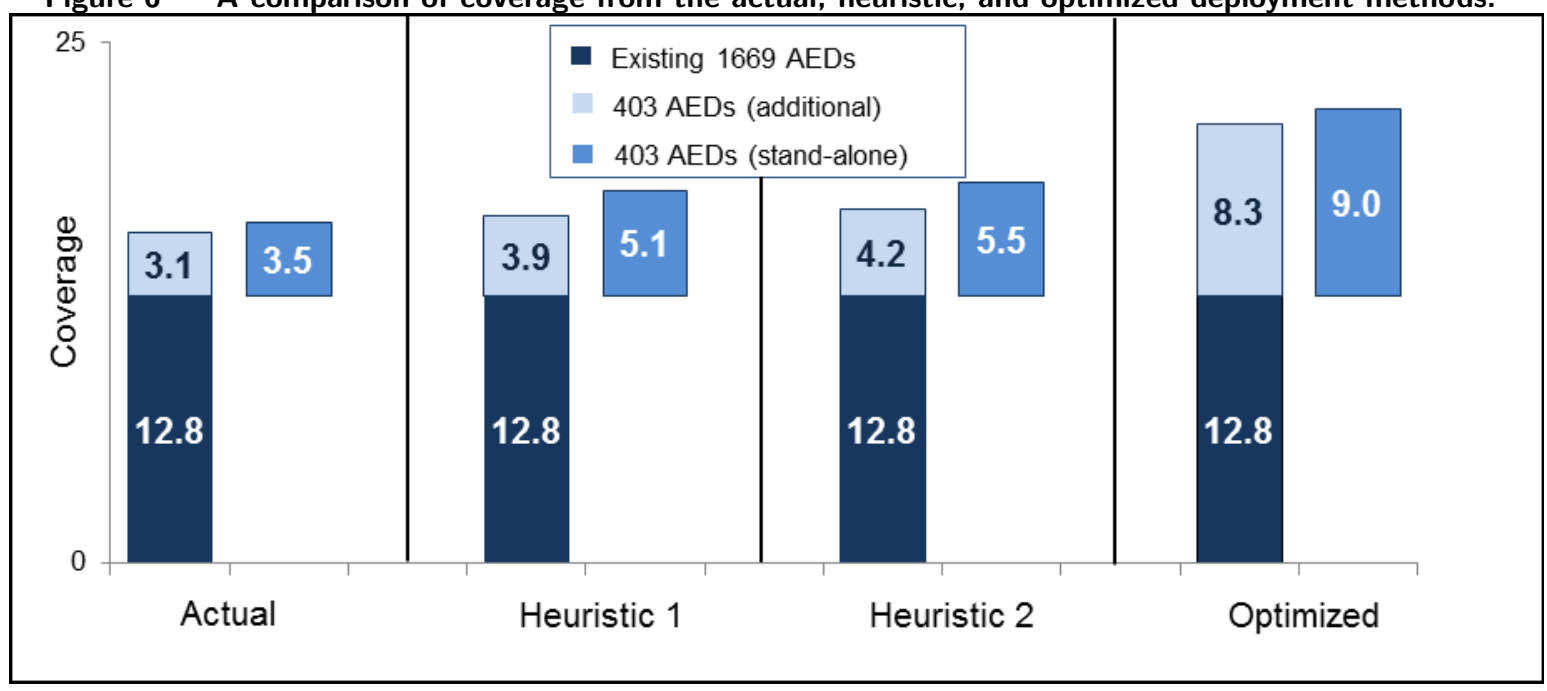

We can also compare these approaches in terms of distance. Figure 7 plots the cumulative distance distribution between every cardiac arrest (over all testing sets) and its closest AED from 
Table 3 Coverage values from actual, heuristic and optimized methods, calculated over 100 testing sets.

\begin{tabular}{|c|c|c|c|c|c|}
\hline AED deployment approach & Mean & Maximum & Minimum & $\mathbf{0 . 1 V a R}$ & Coeff. of Var. \\
\hline \hline Existing 1669 AEDs & 12.8 & 19.2 & 6.8 & 9.5 & $22 \%$ \\
Existing + 403 actual AEDs & 15.9 & 24.3 & 8.2 & 12.2 & $20 \%$ \\
Existing + 403 heuristic 1 AEDs & 16.7 & 25.4 & 8.3 & 12.7 & $21 \%$ \\
Existing + 403 heuristic 2 AEDs & 17.0 & 26.3 & 11.0 & 12.8 & $20 \%$ \\
Existing + 403 optimized AEDs & 21.1 & 30.9 & 12.5 & 16.6 & $18 \%$ \\
\hline
\end{tabular}

optimization, Heuristic 2 and the actual deployment. The results are similar up to about 20 meters, but then optimization outperforms the other approaches as distance increases. There is a five percentage point increase in the number of cardiac arrests within 100 meters of the closest AED compared to the other methods, which corresponds to a $25 \%$ improvement. Heuristic 1 results were very similar to Heuristic 2 and thus are omitted.

Figure 7 Cumulative distance distribution of cardiac arrests to the closest AED.

Because we do not know exactly when the actual AEDs were placed, the previous experiments assumed all 403 AEDs were deployed at once by optimizing the simultaneous placement of all of them (i.e., a single-stage approach). We also considered a multi-stage approach where the AED locations were determined by solving four successive optimization problems, each placing 100-101 AEDs and holding those placed in previous stages fixed. Between the single-stage and multi-stage approaches, we found that the mean coverage differed by less than $0.5 \%$, while the other metrics from Table 3 differed by less than $2 \%$.

\subsection{The Value of Relocating Existing AEDs}

Relocating existing AEDs is not a standard practice for many practical reasons. However it is worthwhile to examine how much improvement would be possible if existing AEDs could be relocated. We reran our models without the existing AED constraint and set $N=1,669$ (the number of existing AEDs as of September 2009). We obtained a mean coverage of 28.4 over the 100 testing sets according to Model 1. For comparison, the mean coverage provided by the same number of existing AEDs was 12.8 (Table 4). To achieve the same coverage level by adding new AEDs, at 
least 900 would need to be added to the existing ones that are currently deployed. Similarly, if there were no existing AEDs in Toronto and AEDs were deployed from scratch, only about 550 would be needed to achieve the same coverage level as the 1,669 existing AEDs. Thus, substantial gains appear to be possible by simply relocating existing AEDs.

Table 4 Coverage values from relocating existing AEDs using optimization, calculated over 100 testing sets.

\begin{tabular}{|c|c|c|c|c|c|}
\hline AED deployment approach & Mean & Maximum & Minimum & 0.1VaR & Coeff. of Var. \\
\hline \hline Existing 1,669 AEDs & 12.8 & 19.2 & 6.8 & 9.5 & $22 \%$ \\
Relocated 1,669 AEDs & 28.4 & 41.6 & 17.7 & 23.8 & $14 \%$ \\
Existing + 900 optimized AEDs & 28.4 & 39.8 & 17.6 & 23.1 & $16 \%$ \\
Relocated 550 AEDs & 12.8 & 20.9 & 7.1 & 9.6 & $22 \%$ \\
\hline
\end{tabular}

\subsection{Map-Based Geographical Analysis of Optimal AED Locations}

To gain some insight and validation of the optimization models, we evaluate the optimal locations from the MCLP model, Model 2 and Model 3 for $N=30$, and the locations of the 1,669 existing registered AEDs, using the geographical information systems software ArcMap 10 (Esri, Redlands, California). Since the Model 3 solution provides a good lower bound on Model 1, the optimal AED locations derived from Model 3 can be thought of as nearly optimal locations for Model 1 in the maps. When a model has multiple optimal solutions, we choose the one that has the most locations in common with the solutions from the other models to facilitate comparisons. To better understand the differences between the models, plotted cardiac arrests are from the training set.

Figure 8 shows the 5,000 cardiac arrests from the training set. As expected, there is a denser concentration of cardiac arrests in and around the downtown area (near the bottom center of the map). Figure 9 shows the optimal solutions of the MCLP model, Models 2 and 3, as well as the locations of the existing registered AEDs. All models place the majority of the AEDs in downtown. Model 3 produces the most concentrated solution, whereas the Model 2 and MCLP solutions are more spread out geographically. Figure 10 illustrates AED locations in downtown. Out of the top 30 locations for AED deployment, Model 3 places 24 in downtown, Model 2 places 21 in downtown, and the MCLP places 16-22 in downtown (the range for is due to multiple optimal solutions).

Figure 11 provides an example to illustrate the difference between decaying coverage models (Models 2 and 3) and a non-decaying coverage model (MCLP); when the true coverage function is decaying, the MCLP can lead to suboptimal solutions. The figure shows a neighborhood intersecting the financial and entertainment districts in downtown Toronto with several cardiac arrest locations. The MCLP model picks an AED location that covers six cardiac arrests within 100 meters, but none within 20 meters, which results in poor coverage as measured by the decaying coverage function. Models 2 and 3 both choose a location that covers four cardiac arrests within 100 meters, providing 
full or nearly full coverage to three of them, resulting in more than double the coverage of the MCLP solution.

Figure 12 illustrates how Model 2 and Model 3 can differ in AED placement. The figure depicts a residential neighborhood in downtown Toronto that has several homeless shelters, the highest number of drug arrests and the fourth highest number of ambulance calls among all 140 neighborhoods in the city (City of Toronto 2011). This neighborhood had the highest number of cardiac arrests over the 4.5 years covered by our dataset. Even though there are two registered AEDs in the area, improved coverage is possible, as evidenced by both Models 2 and 3 placing at least one AED in the vicinity. The map on the left shows the Model 2 solution, which aims to cover the cluster of cardiac arrests in the southeast part of the neighborhood. The map on the right shows the Model 3 solution, which provides coverage to both the cluster in the southeast as well as additional coverage to cardiac arrests that are only minimally covered by the existing registered AEDs. These results support the fact that overlapping coverage areas are beneficial in Model 3 (and Model 1), but not in Model 2 (or the MCLP), which is due to the way the objective functions measure coverage in the models. Thus, Model 2 and the MCLP will always want to spread out AEDs to minimize overlap, whereas Models 1 and 3 will concentrate AED deployment when coverage improvement is possible.

\section{Implications for Public Access Defibrillator Deployment}

Our results suggest that most cardiac arrest coverage is derived from deploying AEDs in urban and often high risk areas, which is supported by the literature. Cardiac arrest incidence is correlated with population density and traffic flow. It has also been shown that out-of-hospital cardiac arrests are more likely to occur during business hours (Brooks et al. 2010). Lower socioeconomic status also tends to correlate with higher cardiac arrest risk (Reinier et al. 2011). All our models tend to concentrate AEDs in and around downtown Toronto, which has the highest daytime population density in the city, and tends to contain more neighborhoods that are socioeconomically depressed. However, note that using population alone in AED location decisions is not sufficient; recall that the solutions generated by optimization outperforms the population-guided methods by a statistically significant amount.

One may argue that simply understanding the demand distribution better will lead to improved performance over the current AED deployment policy. First, we should note that estimating the demand distribution itself is non-trivial in that substantial effort and cost goes into acquiring cardiac arrest data in cities around the world. Second, how AED supply is aligned with demand can take many forms and must be done intelligently. For example, the MCLP essentially aims to match the demand distribution and we demonstrated that the MCLP is suboptimal if true coverage decays with distance or response time. Additionally, lay responder behavior can also influence the 
degree to which AED locations are simply matched to the demand distribution-note that Model 2 does not match the demand distribution as closely as Models 1 and 3 due to the fact that Model 2 does not benefit from overlapping coverage. Thus, although understanding the demand distribution is important to achieve improved performance, other factors such as decaying coverage and lay responder behavior, which we consider, should also be taken into account.

Model 1 considers the case where multiple lay responders at the scene of a cardiac arrest fan out and search for nearby AEDs. We believe this model to be the most suitable to guide deployment decisions and measure coverage, primarily because most AED deployment tends to be in dense urban areas, which are more likely to have multiple bystanders in a cardiac arrest emergency. Additional realistic considerations can be introduced in this model through appropriate use of the $p_{i j}$ parameter. For instance, $p_{i j}$ can be reduced in rural areas where the probability of having a nearby lay responder witness a cardiac arrest is less than in dense urban areas. This situation could be modeled with a coverage function that is shifted downwards. The values of $p_{i j}$ can also be adjusted for each potential AED location $i$ based on the accessibility of the location as influenced by locked doors, multiple floors, and hours of operation.

\subsection{Lay Responder Behavior}

Since little is known about lay responder behavior in a cardiac arrest emergency, modeling such behavior is challenging. Instead, Models 2 and 3 provide "bounds" on AED retrieval in the singleresponder case. The $50 \%$ difference in coverage (Figure 5) between those two models can be interpreted as the improvement potential of complementary initiatives aimed at optimizing lay responder behavior during AED retrieval. For example, efforts to improve AED registration and integration with 911, public awareness of AEDs, and signage in public buildings may result in AEDs being found more easily. The behavior of lay responders is important; indeed, it is straightforward to show that shifting lay responder behavior from Model 2 to Model 3 is equivalent to adding 250-300 AEDs to the current network. Additionally, smartphone applications like PulsePoint (PulsePoint 2012) can help 911 dispatchers recruit and guide lay responders in an emergency situation to the closest AED. Such applications have the potential to extend the coverage radius of public AEDs by encouraging responders close to an AED to make a one-way trip to the patient, instead of having a responder close to the patient making a round-trip. Note that optimization models can also be a policy evaluation tool, to measure the potential impact of technological innovations that can increase the effective radius of public AEDs (Siddiq et al. 2013).

\subsection{Coverage Versus Survival}

In this study, we focus on coverage as the primary performance measure with the understanding that it serves as a proxy for survival. Survival is complicated because it depends on many factors 
beyond response time such as age and co-morbidities like heart disease, smoking, and obesity. However, we offer some insight on the potential of our framework to improve survival. We use the simplified logistic regression survival model of Valenzuela et al. (1997) and make the following assumptions: survival only depends on time to defibrillation and time to CPR; a lay responder who performs CPR is also willing to search for and use an AED, and finds the closest AED; when delivered, lay responder $\mathrm{CPR}$ is delivered right after collapse; in the absence of lay responder $\mathrm{CPR}$, paramedics start CPR as soon as the ambulance arrives; it takes lay responders 1.5 minutes and paramedics 1 minute to set up an AED (Gundry et al. 1999); average ambulance response time is 6 minutes and $35 \%$ of lay responders provide CPR (from our data); the threshold for the survival function is chosen to achieve $80 \%$ sensitivity (survivors correctly classified). With these assumptions, we revisit the comparison from Section 6.2 between the 403 actual AED locations versus 403 locations determined through optimization. We estimate that the optimized deployment saves $9-11$ lives per year, which is a $28-55 \%$ increase compared to the actual deployment (6-8 lives saved). Although there are many assumptions that go into these calculations, we believe these results indicate potential for survival improvement using optimization.

\subsection{Cost-Effectiveness of Optimizing AED Deployment}

Several studies have evaluated the cost-effectiveness of public access defibrillation programs. The typical range is between (in 2015 dollars) $\$ 60,000$ - $\$ 87,000$ per quality-adjusted life year (QALY), with variation due to the locations considered for AED deployment, assumed AED usage rate, costs, etc. (Nichol et al. 1998, Walker et al. 2003, Nichol et al. 2003, Folke et al. 2009). Next, we return to the example of placing 403 AEDs using optimization and estimate the associated cost-effectiveness of that strategy. We make the following assumptions: an AED costs $\$ 1,800$; maintenance costs are $\$ 100$ every two years and every time an AED is used during its life of eight years (Physio-control Inc. 2010); a hospital stay is $\$ 91,960$ for a patient discharged alive and $\$ 4,160$ for a patient who dies in hospital (Nichol et al. 2003); for every one cardiac arrest patient discharged alive 3.3 patients die in hospital (from our data); annual medical costs for survivors are $\$ 15,740$ (Folke et al. 2009); the optimization approach saves 10 lives on average per year; life expectancy of survivors post-arrest is 6.2 years (Walker et al. 2003); the mean quality of life of a survivor is 0.72 (Nichol et al. 1999). All dollar amounts above represent 2015 U.S. dollars and both costs and benefits are discounted at $3 \%$ in accordance with World Health Organization guidelines (Edejer et al. 2003). The total cost of placing 403 AEDs optimally is approximately $\$ 2$ million, of which only $6 \%$ is related to the AED costs. The cost per QALY is $\$ 50,150$. This result suggests that optimization improves the cost-effectiveness of public AED deployment - the previous cost-effectiveness studies evaluated programs that deployed AEDs without optimization. The most widely accepted benchmark for costeffectiveness in medical interventions is $\$ 50,000$ per QALY (Mason et al. 1993), which translates 
to roughly $\$ 81,000$ in 2015 dollars. A recent study suggests using $\$ 100,000-\$ 150,000$ as a more up-to-date benchmark (Neumann et al. 2014). Either way, our results suggest that optimizing AED deployment is a cost-effective intervention. Note that relocating existing AEDs in lieu of adding new ones may result in significant savings - up to $\$ 1.6$ million based on the analysis in Section 6.3, which showed that relocating the existing 1,669 AEDs using optimization is equivalent to adding 900 new AEDs.

\subsection{The Need for a Central Decision Maker}

Identifying good locations to place AEDs is one critical component of a PAD program and of the larger effort to increase survival from out-of-hospital cardiac arrest. Our results demonstrate that this process can benefit from using an optimization-driven framework to guide decision making. Currently there is no central decision maker for AED deployment. In Canada, the closest such entity is the HSFC (or the federal or provincial government when they provide targeted funding for certain sites). We envision that the provincial arms of HSFC, working with municipal representatives including local EMS and PAD programs, would be best positioned to make decisions in a centralized fashion. Such a decision maker could actively target priority areas for AED deployment, instead of waiting for applications from the community. These neighborhoods would then be evaluated in partnership with the local community and businesses to determine precise locations to place AEDs, accounting for factors such as traffic patterns and building accessibility. Having a centralized decision maker, supported by our modeling framework, would ensure that high risk areas are not overlooked and that AED deployment is managed in a coordinated fashion.

\section{Conclusions}

Automated external defibrillators are potentially life-saving devices for victims of cardiac arrest. AEDs need to be placed intelligently in public settings so that they are likely to be used by bystanders. In this paper, we develop the first comprehensive data-driven framework to support public AED location decisions, leveraging optimization and statistical models. We apply our methodology to real data from Toronto, Canada. Our results demonstrate that optimizing AED locations can result in a substantial improvement in coverage compared to the current approach to AED deployment as well as two population-guided heuristics. Similarly, relocating existing AEDs can have a significant impact on coverage and cost. We provide several practical insights for public AED programs and demonstrate that optimizing AED deployment is a cost-effective intervention.

\section{Appendix. Proofs}

The following lemmata are used in the proof of Proposition 1. Proofs are straightforward and are omitted. 
Figure 85000 cardiac arrest locations comprising the training set, Toronto.

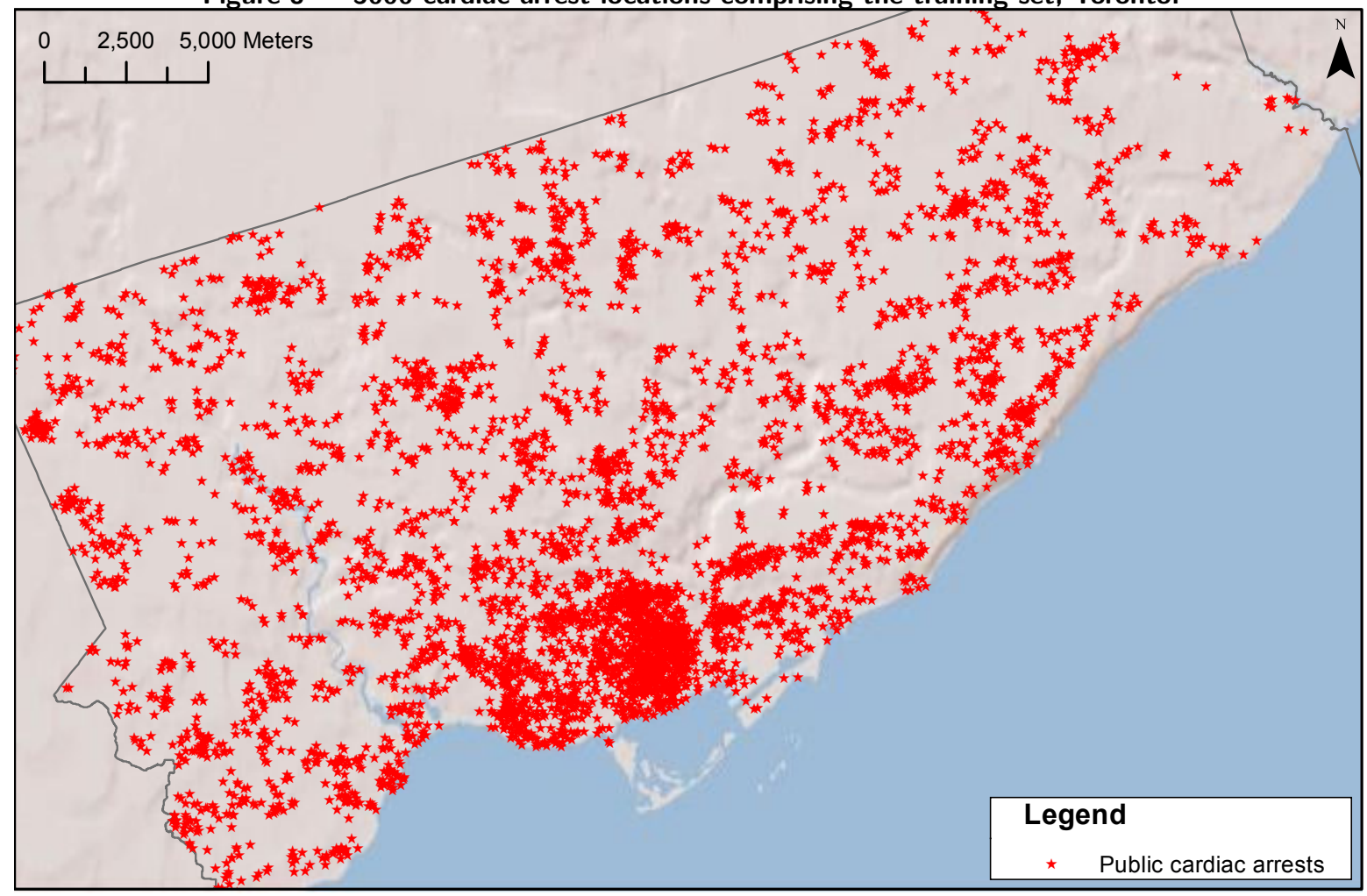

Figure 9 Optimal and existing AED locations, Toronto.

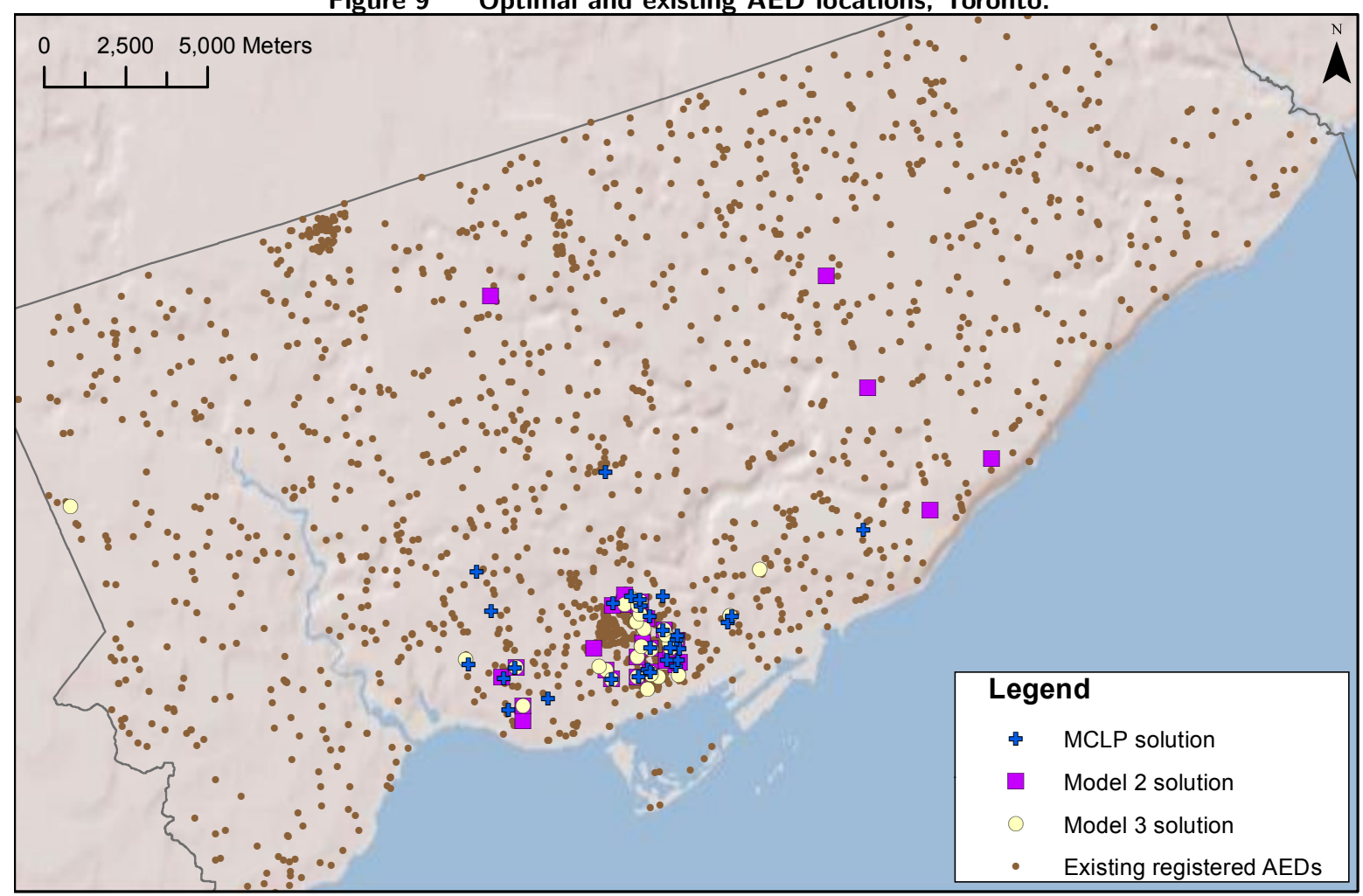


Figure 10 Optimal AED locations, downtown Toronto.

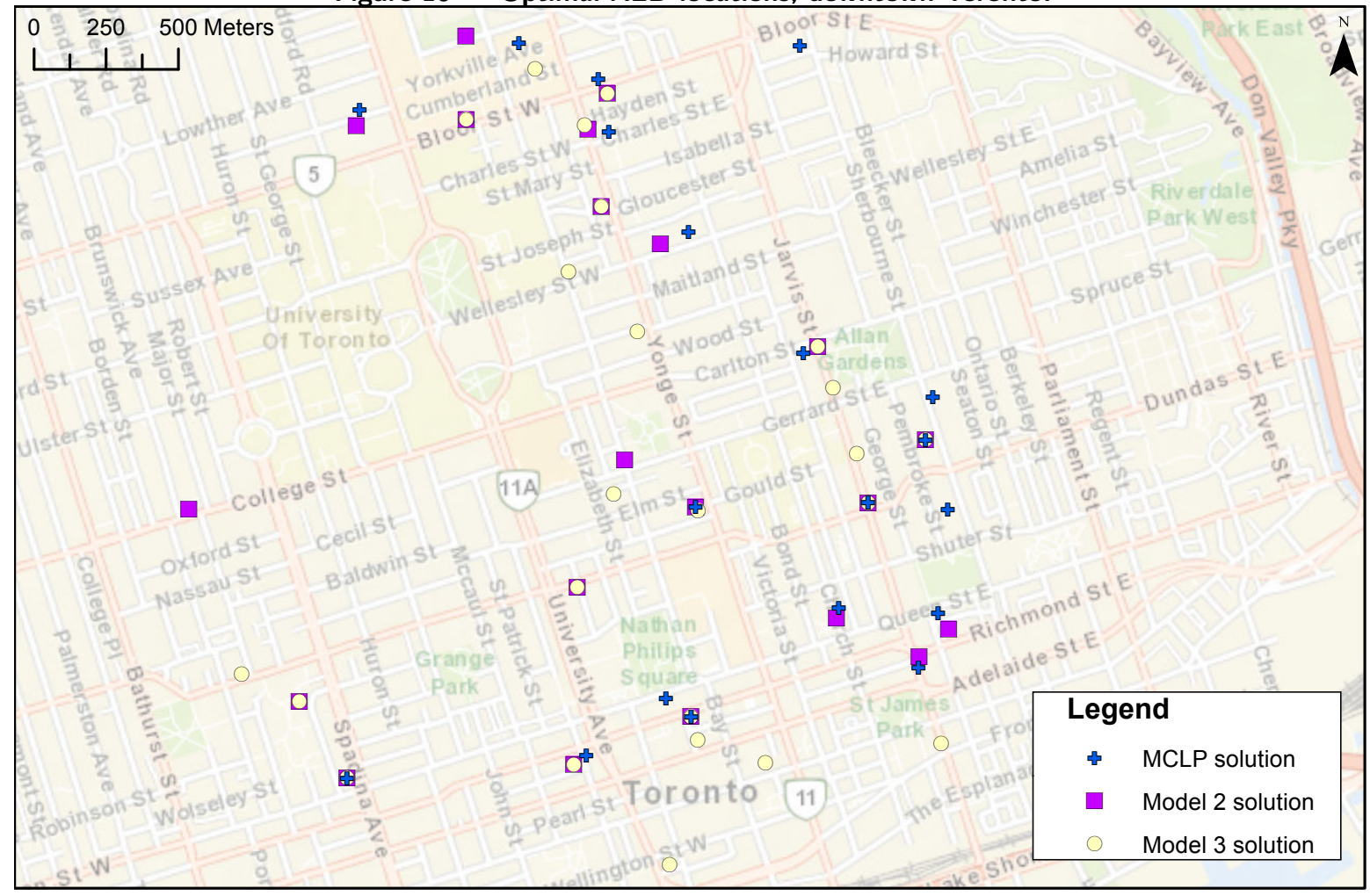

LEMma 1. A feasible solution to formulation (12) is also feasible for formulation (13).

LEMma 2. An optimal solution to formulation (13) is feasible for formulation (12).

Proof of Proposition 1. For the forward direction, let $(\mathbf{r}, \mathbf{z}, \mathbf{y})$ be an optimal solution for formulation (13), which is also feasible for formulation (12) by Lemma 2. Suppose to the contrary that there is a feasible solution $(\overline{\mathbf{r}}, \overline{\mathbf{z}}, \overline{\mathbf{y}})$ to formulation (12) such that $\sum_{j \in J} \bar{r}_{j}>\sum_{j \in J} r_{j}$. By Lemma $1,(\overline{\mathbf{r}}, \overline{\mathbf{z}}, \overline{\mathbf{y}})$ is also feasible for formulation (13), which contradicts the optimality of $(\mathbf{r}, \mathbf{z}, \mathbf{y})$ for formulation (13).

The reverse direction is similar and omitted.

Proof of Proposition 2. Let $\mathbf{y}$ be feasible for both problems and let $I_{j}^{1}=\left\{i \in I \mid p_{i j}>0\right.$ and $\left.y_{i}=1\right\}$. For a given $j$, constraint (15c) implies $\sum_{i \in I_{j}^{1}} w_{i j}=\sum_{i \in I_{j}} w_{i j} \leq 1$, since $w_{i j}$ is forced to 0 if $y_{i}=0$ by constraint (15b). If $I_{j}^{1} \neq \emptyset$, at most one $w_{i j}$ will be equal to one. At optimality, given $\mathbf{y}$, the optimal values of $w_{i j}$ are

$$
w_{i j}=\left\{\begin{array}{l}
1, \text { if } I_{j}^{1} \neq \emptyset \text { and } i \in \arg \max _{i^{\prime} \in I_{j}^{1}}\left(p_{i^{\prime} j}\right), \\
0, \text { otherwise. }
\end{array}\right.
$$

Given these values of $w_{i j}$, the objective function of problem (15) becomes

$$
\sum_{j \in J} \sum_{i \in I_{j}} p_{i j} w_{i j}=\sum_{j \in J} \max _{i \in I_{j}^{1}}\left(p_{i j}\right)=\sum_{j \in J} \max _{i \in I_{j}}\left(p_{i j} y_{i}\right)=\sum_{j \in J} \max _{i \in I}\left(p_{i j} y_{i}\right)=\sum_{j \in J} r_{j},
$$

which is the objective function of problem (14). 


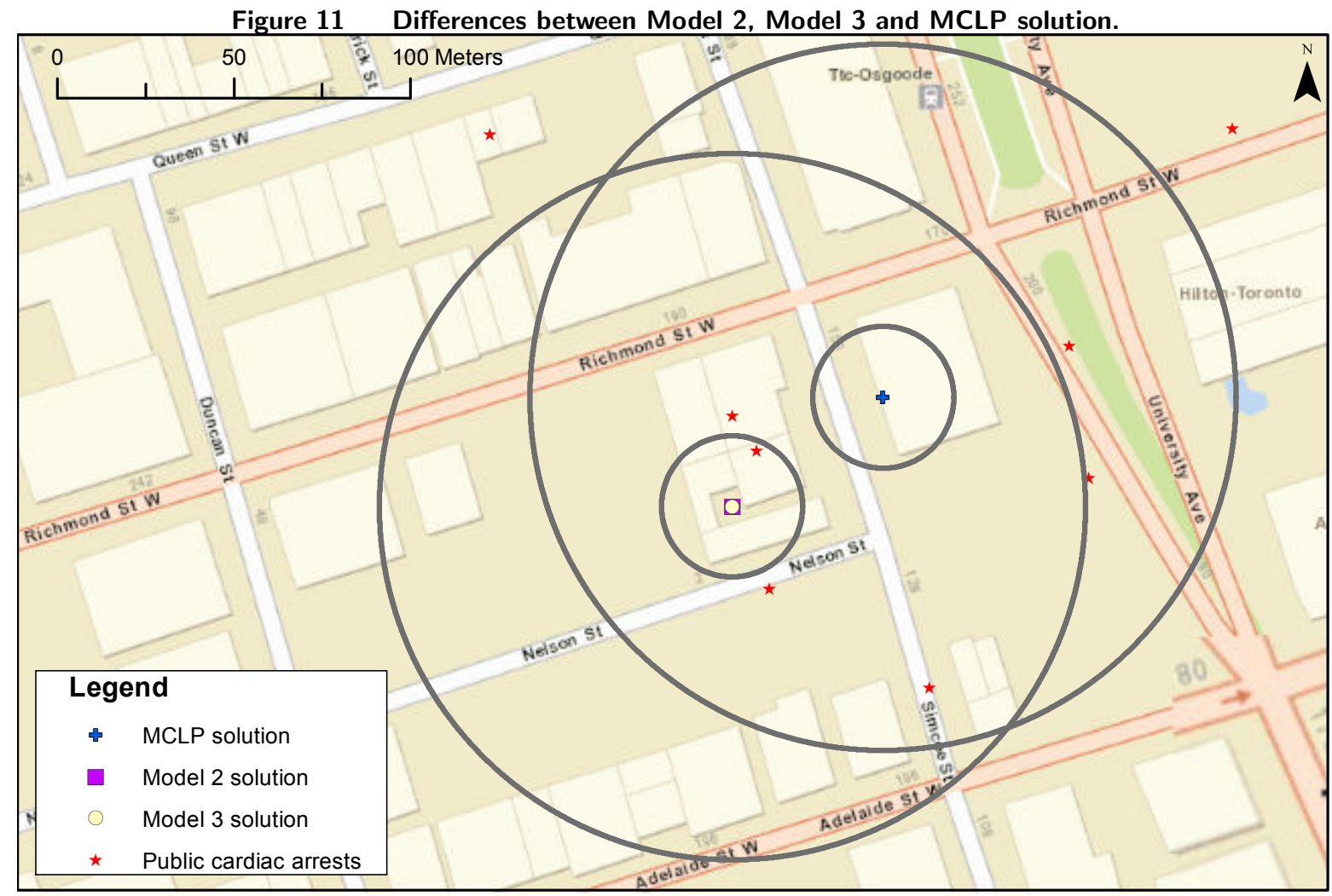

Note. The cardiac arrests come from the training set

Proof of Proposition 3. Let $I_{j}=\left\{i \in I \mid p_{i j}>0\right\}$ and $I_{j}^{1}=\left\{i \in I \mid p_{i j}>0\right.$ and $\left.y_{i}=1\right\}$. Also, for each $j \in J$, let $r_{j}^{k}$ be the variable $r_{j}$ in Model $k,(k=1,2,3)$ and $x_{j}$ be as defined in the MCLP (1). For any $j$, if $I_{j}^{1}=\emptyset$, then $r_{j}^{1}=r_{j}^{2}=r_{j}^{3}=x_{j}=0$ for any value of $p_{i j}$. Suppose $I_{j}^{1} \neq \emptyset$. In the MCLP, $x_{j}=1$. For the other models,

$$
\begin{gathered}
r_{j}^{1}=1-\prod_{i \in I}\left(1-p_{i j} y_{i}\right)=1-\prod_{i \in I_{j}^{1}}\left(1-p_{i j}\right)=1-\left(1-p_{k j}\right) \prod_{i \in I_{j}^{1} \backslash\{k\}}\left(1-p_{i j}\right) \geq p_{k j}, \quad \forall k \in I_{j}^{1} \\
r_{j}^{2}=\min _{i \in I_{j}^{1}} p_{i j} y_{i}=\min _{i \in I_{j}^{1}} p_{i j} \\
r_{j}^{3}=\max _{i \in I} p_{i j} y_{i}=\max _{i \in I_{j}^{1}} p_{i j} .
\end{gathered}
$$

Thus, $1 \geq r_{j}^{1} \geq r_{j}^{3} \geq r_{j}^{2}$. These inequalities hold for any feasible $\mathbf{y}$, and since a feasible $\mathbf{y}$ for one formulation is feasible for all of them, $Z_{M C L P}^{\mathbf{y}} \geq Z_{1}^{\mathbf{y}} \geq Z_{3}^{\mathbf{y}} \geq Z_{2}^{\mathbf{y}}$. Moreover, if $p_{i j} \in\{0,1\}$ for all $i \in I, j \in J$, then

$$
\begin{gathered}
r_{j}^{1}=1-\prod_{i \in I}\left(1-p_{i j} y_{i}\right)=1-\prod_{i \in I_{j}}\left(1-y_{i}\right)=1 \\
r_{j}^{2}=\min _{i \in I_{j}^{1}} p_{i j} y_{i}=1 \\
r_{j}^{3}=\max _{i \in I} p_{i j} y_{i}=1
\end{gathered}
$$

since $I_{j}^{1} \neq \emptyset$. Hence, $r_{j}^{1}=r_{j}^{2}=r_{j}^{3}=x_{j}=1$ and by the same $\operatorname{argument}$ above, $Z_{M C L P}^{\mathrm{y}}=Z_{1}^{\mathrm{y}}=Z_{3}^{\mathrm{y}}=Z_{2}^{\mathrm{y}}$.

Proof of Theorem 1. Let $Z_{k}^{\mathbf{y}}$ be the objective function value of Model $k, k \in\{1,2,3, M C L P\}$, given a feasible $\mathbf{y}$. Also, let $\mathbf{y}^{k}$ be the optimal $\mathbf{y}$ in Model $k, k \in\{1,2,3, M C L P\} . Z_{M C L P} \geq Z_{M C L P}^{\mathbf{y}^{1}}, Z_{1} \geq Z_{1}^{\mathbf{y}^{3}}$, and $Z_{3} \geq Z_{3}^{\mathrm{y}^{2}}$, by optimality. $Z_{M C L P}^{\mathrm{y}^{1}} \geq Z_{1}, Z_{1}^{\mathrm{y}^{3}} \geq Z_{3}$, and $Z_{3}^{\mathrm{y}^{2}} \geq Z_{2}$, by Proposition 3 . Therefore,

$$
Z_{M C L P} \geq Z_{1} \geq Z_{3} \geq Z_{2} \text {. }
$$


Figure 12 Differences between Model 2 and Model 3.

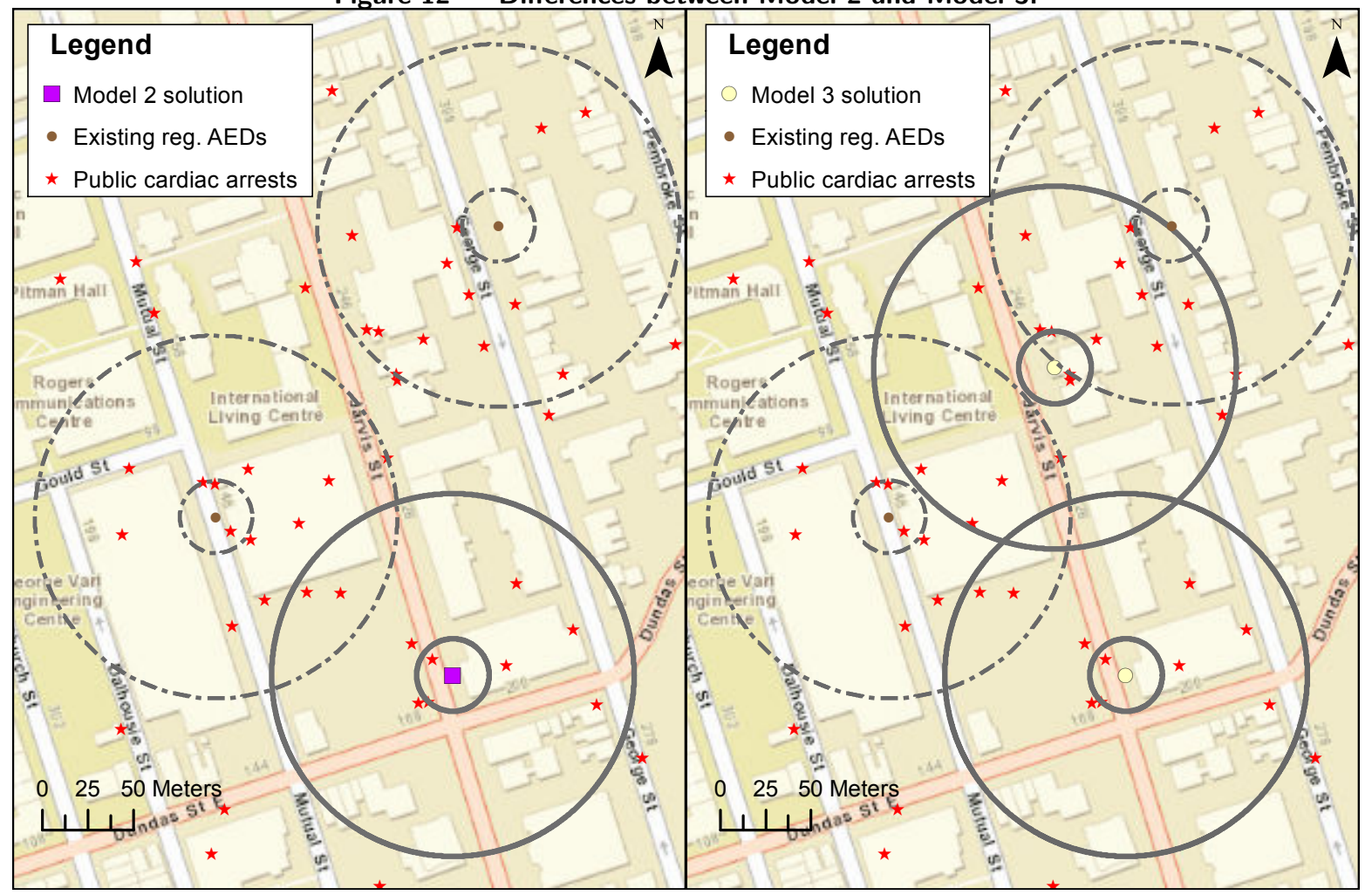

Note. The cardiac arrests come from the training set

In the special case that $p_{i j} \in\{0,1\}$ for all $i \in I, j \in J, Z_{1}^{\mathbf{y}^{M C L P}}=Z_{M C L P}, Z_{3}^{\mathbf{y}^{1}}=Z_{1}$, and $Z_{2}^{\mathbf{y}^{3}}=Z_{3}$, by Proposition 3. $Z_{1} \geq Z_{1}^{\mathbf{y}^{M C L P}}, Z_{3} \geq Z_{3}^{\mathbf{y}^{1}}$, and $Z_{2} \geq Z_{2}^{\mathrm{y}^{3}}$, by optimality. Therefore, $Z_{M C L P} \leq Z_{1} \leq Z_{3} \leq Z_{2}$. Together with equation (17), this implies $Z_{M C L P}=Z_{1}=Z_{3}=Z_{2}$.

\section{Acknowledgment}

We sincerely thank department editor Dimitris Bertsimas, the associate editor and three referees for their helpful comments and guidance throughout the review process. We thank Sebastian Jaimungal and Shane Henderson for helpful discussions on kernel density estimation. We are grateful to our medical collaborators Steve Brooks and Laurie Morrison for their valuable feedback and access to the cardiac arrest data. Lastly, we acknowledge the City Planning Division of the City of Toronto for providing data on public buildings, Toronto EMS for providing data on public access AEDs, and HPCVL for providing computing resources.

\section{References}

Adams, W.P., H.D. Sherali. 1986. A tight linearization and an algorithm for zero-one quadratic programming problems. Management Sci. 32(10) 1274-1290.

Aufderheide, T., M.F. Hazinski, G. Nichol, S.S. Steffens, A. Buroker, R. McCune, E. Stapleton, V. Nadkarni, J. Potts, R.R. Ramirez, et al. 2006. Community lay rescuer automated external defibrillation programs. Circulation 113(9) 1260-1270. 
Becker, L., M. Eisenberg, C. Fahrenbruch, L. Cobb. 1998. Public locations of cardiac arrest: Implications for public access defibrillation. Circulation 97(21) 2106.

Berman, O., D. Krass, Z. Drezner. 2003. The gradual covering decay location problem on a network. Eur. J. Oper. Res. 151(3) 474-480.

Botev, Z.I., J.F. Grotowski, D.P. Kroese. 2010. Kernel density estimation via diffusion. Ann. Stat. 38(5) $2916-2957$.

Brooks, S.C., J.H. Hsu, S.K. Tang, R. Jeyakumar, T.C.Y. Chan. 2013. Determining risk for out-of-hospital cardiac arrest by location type in a Canadian urban setting to guide future public access defibrillator placement. Ann. Emerg. Med. 61(5) 530-538.

Brooks, S.C., R.H. Schmicker, T.D. Rea, T.P. Aufderheide, D.P. Davis, L.J. Morrison, R. Sahni, G.K. Sears, D.E. Griffiths, G. Sopko, et al. 2010. Out-of-hospital cardiac arrest frequency and survival: Evidence for temporal variability. Resuscitation $\mathbf{8 1}(2)$ 175-181.

Caffrey, S.L., P.J. Willoughby, P.E. Pepe, L.B. Becker. 2002. Public use of automated external defibrillators. N. Engl. J. Med. 347(16) 1242-1247.

Chan, T.C.Y., H. Li, G. Lebovic, S.K. Tang, J.Y.T. Chan, H.C.K. Cheng, L.J. Morrison, S.C. Brooks. 2013. Identifying locations for public access defibrillators using mathematical optimization. Circulation $127(17)$ 1801-1809.

Chan, T.C.Y., Z.M. Shen, A.A. Siddiq. 2015. Robust facility location under demand location uncertainty. Submitted, available on arXiv preprint arXiv:1507.04397.

Church, R., C. ReVelle. 1974. The maximal covering location problem. Pap. Reg. Sci. 32(1) 101-118.

Church, R.L., K.L. Roberts. 1983. Generalized coverage models and public facility location. Pap. Reg. Sci. 53(1) $117-135$.

City of Toronto. 2010. Toronto employment survey 2009. http://www.toronto.ca/demographics/pdf/ survey2009.pdf. [Online; accessed 7-May-2013].

City of Toronto. 2011. Wellbeing toronto. http://map.toronto.ca/wellbeing. [Online; accessed 7-May-2013].

Culley, L.L., T.D. Rea, J.A. Murray, B. Welles, C.E. Fahrenbruch, M. Olsufka, M.S. Eisenberg, M.K. Copass. 2004. Public access defibrillation in out-of-hospital cardiac arrest. Circulation 109(15) 1859-1863.

Dao, T.H.D., Y. Zhou, J.C. Thill, E. Delmelle. 2011. Spatio-temporal location modeling in a 3d indoor environment: The case of AEDs as emergency medical devices. Int. J. Geogr. Inf. Sci. 26(3) 469-494.

Daskin, M.S. 1983. A maximum expected covering location model: Formulation, properties and heuristic solution. Transportation Sci. 17(1) 48.

Daskin, M.S. 2008. What you should know about location modeling. Nav. Res. Log. 55(4) 283-294.

Daskin, M.S., L.K. Dean. 2005. Location of health care facilities. Operations Research and Health Care: A Handbook of Methods and Applications. Springer, 43-76. 
De Maio, V.J., I.G. Stiell, G.A. Wells, D.W. Spaite. 2003. Optimal defibrillation response intervals for maximum out-of-hospital cardiac arrest survival rates. Ann. Emerg. Med. 42(2) 242-250.

Edejer, T., R. Baltussen, T. Adam, R. Hutubessy, A. Acharya, D.B. Evans, C.J.L. Murray. 2003. Making Choices in Health: WHO Guide to Cost-Effectiveness Analysis. World Health Organization.

Engdahl, J., J. Herlitz. 2005. Localization of out-of-hospital cardiac arrest in Göteborg 1994-2002 and implications for public access defibrillation. Resuscitation 64(2) 171-175.

Erkut, E., A. Ingolfsson, G. Erdoğan. 2008. Ambulance location for maximum survival. Nav. Res. Log. 55(1) $42-58$.

Fallah, H., A.N. Sadigh, M. Aslanzadeh. 2009. Covering problem. Facility Location: Concepts, Models, Algorithms and Case Studies. Springer, 145-176.

Fedoruk, J.C., W.L. Currie, M. Gobet. 2002. Locations of cardiac arrest: Affirmation for community public access defibrillation (PAD) program. Prehosp. Disaster Med. 17(4) 202-205.

Fisher, R.A. 1921. On the "probable error" of a coefficient of correlation deduced from a small sample. Metron $13-32$.

Folke, F., F.K. Lippert, S.L. Nielsen, G.H. Gislason, M.L. Hansen, T.K. Schramm, R. Sørensen, E.L. Fosbøl, S.S. Andersen, S. Rasmussen, et al. 2009. Location of cardiac arrest in a city center: Strategic placement of automated external defibrillators in public locations. Circulation 120(6) 510-517.

Gendreau, M., G. Laporte, F. Semet. 1997. Solving an ambulance location model by tabu search. Location Sci. 5(2) 75-88.

Go, A.S., D. Mozaffarian, V.L. Roger, E.J. Benjamin, J.D. Berry, M.J. Blaha, S. Dai, E.S. Ford, C.S. Fox, S. Franco, et al. 2013. Heart disease and stroke statistics-2014 update: A report from the American Heart Association. Circulation 129 e206.

Gratton, M., D.J. Lindholm, J.P. Campbell. 1999. Public-access defibrillation: Where do we place the AEDs? Prehosp. Emerg. Care 3(4) 303-305.

Gundry, J.W., K.A. Comess, F.A. DeRook, D. Jorgenson, G.H. Bardy. 1999. Comparison of naive sixthgrade children with trained professionals in the use of an automated external defibrillator. Circulation 100(16) 1703.

Hallstrom, A.P., J.P. Ornato, M. Weisfeldt, A. Travers, J. Christenson, M.A. McBurnie, R. Zalenski, L.B. Becker, E.B. Schron, M. Proschan. 2004. Public-access defibrillation and survival after out-of-hospital cardiac arrest. N. Engl. J. Med. 351(7) 637-646.

Hazinski, M.F., A.H. Idris, R.E. Kerber, A. Epstein, D. Atkins, W. Tang, K. Lurie. 2005. Lay rescuer automated external defibrillator ("public access defibrillation") programs. Circulation 111(24) 33363340.

Heart and Stroke Foundation of Canada. 2013. Statistics: Cardiac arrest. http://www.heartandstroke. com/site/c.ikIQLcMWJtE/b.3483991/k.34A8/Statistics.htm. [Online; accessed 1-April-2015]. 
Karasakal, O., E.K. Karasakal. 2004. A maximal covering location model in the presence of partial coverage. Comput. Oper. Res. 31(9) 1515-1526.

Larsen, M.P., M.S. Eisenberg, R.O. Cummins, A.P. Hallstrom. 1993. Predicting survival from out-of-hospital cardiac arrest: A graphic model. Ann. Emerg. Med. 22(11) 1652-1658.

Lee, Y., H.D. Sherali, I. Kwon, S. Kim. 2006. A new reformulation approach for the generalized partial covering problem. Nav. Res. Log. 53(2) 170-179.

Lerner, E.B., R.J. Fairbanks, M.N. Shah. 2005. Identification of out-of-hospital cardiac arrest clusters using a geographic information system. Acad. Emerg. Med. 12(1) 81-84.

Mandell, M.B., L.R. Becker. 1996. A model for locating automatic external defibrillators. Socioecon. Plann. Sci. 30(1) 51-66.

Mason, J., M. Drummond, G. Torrance. 1993. Some guidelines on the use of cost effectiveness league tables. BMJ 306(6877) 570-572.

Mell, H.K., M.R. Sayre. 2008. Public access defibrillators and fire extinguishers: Are comparisons reasonable? Prog. Cardiovasc. Dis. 51(3) 204-212.

Morrison, L.J., G. Nichol, T.D. Rea, J. Christenson, C.W. Callaway, S. Stephens, R.G. Pirrallo, D.L. Atkins, D.P. Davis, A.H. Idris, et al. 2008. Rationale, development and implementation of the resuscitation outcomes consortium epistry-cardiac arrest. Resuscitation 78(2) 161-169.

Myers, D.C., M. Mohite. 2008. Locating automated external defibrillators in a university community. J. Oper. Res. Soc. 60(6) 869-872.

Neumann, P.J., J.T. Cohen, M.C. Weinstein. 2014. Updating cost-effectivenessthe curious resilience of the \$50,000-per-qaly threshold. N. Engl. J. Med. 371(9) 796-797.

Nichol, G., A.P. Hallstrom, J.P. Ornato, B. Riegel, I.G. Stiell, T. Valenzuela, G.A. Wells, R.D. White, M.L. Weisfeldt. 1998. Potential cost-effectiveness of public access defibrillation in the United States. Circulation 97(13) 1315-1320.

Nichol, G., I.G. Stiell, P. Hebert, G.A. Wells, K. Vandemheen, A. Laupacis. 1999. What is the quality of life for survivors of cardiac arrest? A prospective study. Acad. Emerg. Med. 6(2) 95-102.

Nichol, G., E. Thomas, C.W. Callaway, J. Hedges, J.L. Powell, T.P. Aufderheide, T. Rea, R. Lowe, T. Brown, J. Dreyer, et al. 2008. Regional variation in out-of-hospital cardiac arrest incidence and outcome. JAMA 300(12) 1423-1431.

Nichol, G., T. Valenzuela, D. Roe, L. Clark, E. Huszti, G.A. Wells. 2003. Cost effectiveness of defibrillation by targeted responders in public settings. Circulation 108(6) 697-703.

Page, R.L., J.A. Joglar, R.C. Kowal, J.D. Zagrodzky, L.L. Nelson, K. Ramaswamy, S.J. Barbera, M.H. Hamdan, D.K. McKenas. 2000. Use of automated external defibrillators by a U.S. airline. N. Engl. J. Med. 343(17) 1210-1216. 
Physio-control Inc. 2010. Lifepak CR Plus AED brochure. http://www.physio-control.com/products/ defibrillators/documents.aspx?id=3355. [Online; accessed 24-March-2015].

PulsePoint. 2012. PulsePoint foundation. http://pulsepoint.org/app/. [Online; accessed 7-May-2013].

Rauner, M.S., N. Bajmoczy. 2003. How many AEDs in which region? An economic decision model for the Austrian Red Cross. Eur. J. Oper. Res. 150(1) 3-18.

Reinier, K., E. Thomas, D.L. Andrusiek, T.P. Aufderheide, S.C. Brooks, C.W. Callaway, P.E. Pepe, T.D. Rea, R.H. Schmicker, C. Vaillancourt, et al. 2011. Socioeconomic status and incidence of sudden cardiac arrest. CMAJ 183(15) 1705-1712.

Sasson, C., M.T. Cudnik, A. Nassel, H. Semple, D.J. Magid, M. Sayre, D. Keseg, J.S. Haukoos, C.R. Warden. 2012. Identifying high-risk geographic areas for cardiac arrest using three methods for cluster analysis. Acad. Emerg. Med. 19(2) 139-146.

Sasson, C., C.C. Keirns, D. Smith, M. Sayre, M. Macy, W. Meurer, B.F. McNally, A.L. Kellermann, T.J. Iwashyna, CARES Study Group. 2010. Small area variations in out-of-hospital cardiac arrest: Does the neighborhood matter? Ann. Intern. Med. 153(1) 19-22.

Scott, D.W. 2009. Multivariate Density Estimation: Theory, Practice, and Visualization, vol. 383. Wiley.

Sheather, S.J., M.C. Jones. 1991. A reliable data-based bandwidth selection method for kernel density estimation. J. Roy. Stat. Soc B Met. 683-690.

Sherali, H.D., S.I. Kim, E.L. Parrish. 1991. Probabilistic partial set covering problems. Nav. Res. Log. 38(1) $41-51$.

Shrout, P.E., J.L. Fleiss. 1979. Intraclass correlations: Uses in assessing rater reliability. Psychol. Bull. 86(2) 420.

Siddiq, A.A., S.C. Brooks, T.C.Y Chan. 2013. Modeling the impact of public access defibrillator range on public location cardiac arrest coverage. Resuscitation 84(7) 904-909.

Soo, L., N. Huff, D. Gray, J.R. Hampton. 2001. Geographical distribution of cardiac arrest in Nottinghamshire. Resuscitation 48(2) 137-147.

Toregas, C., R. Swain, C. ReVelle, L. Bergman. 1971. The location of emergency service facilities. Oper. Res. 19(6) 1363-1373.

Townsend, P. 1987. Deprivation. J. Soc. Policy 16(2) 125-146.

Valenzuela, T.D., D.J. Roe, S. Cretin, D.W. Spaite, M.P. Larsen. 1997. Estimating effectiveness of cardiac arrest interventions: A logistic regression survival model. Circulation 96(10) 3308-3313.

Valenzuela, T.D., D.J. Roe, G. Nichol, L.L. Clark, D.W. Spaite, R.G. Hardman. 2000. Outcomes of rapid defibrillation by security officers after cardiac arrest in casinos. N. Engl. J. Med. 343(17) 1206-1209.

Waalewijn, R.A., R. de Vos, J.G.P. Tijssen, R.W. Koster. 2001. Survival models for out-of-hospital cardiopulmonary resuscitation from the perspectives of the bystander, the first responder, and the paramedic. Resuscitation 51(2) 113-122. 
Walker, A., J.M. Sirel, A.K. Marsden, S.M. Cobbe, J.P. Pell. 2003. Cost effectiveness and cost utility model of public place defibrillators in improving survival after prehospital cardiopulmonary arrest. $B M J$ 327(7427) 1316.

Wand, M.P., M.C. Jones. 1995. Kernel Smoothing, vol. 60. Chapman \& Hall/CRC. 